\title{
1 The epigenetic evolution of gliomas is determined by their IDH1 mutation status and treatment regimen
}

4 Tathiane M Malta ${ }^{1,29}$, Thais S Sabedot ${ }^{2,29}$, Indrani Datta ${ }^{2,30}$, Luciano Garofano ${ }^{3,30}$, Wies Vallentgoed ${ }^{4,30}$, 5 Frederick S Varn ${ }^{5,30}$, Kenneth Aldape ${ }^{6}$, Fulvio D'Angelo ${ }^{3}$, Spyridon Bakas ${ }^{7,8,9}$, Jill S Barnholtz-Sloan ${ }^{6}$, Hui

$6 \mathrm{~K} \mathrm{Gan}^{10}$, Mohammad Hasanain ${ }^{3}$, Ann-Christin Hau ${ }^{11}$, Kevin C Johnson ${ }^{5}$, Mustafa Khasraw ${ }^{12}$, Emre 7 Kocakavuk $^{5}$, Mathilde CM Kouwenhoven ${ }^{13}$, Simona Migliozzi ${ }^{3}$, Simone P Niclou ${ }^{11}$, Johanna M Niers ${ }^{13}$, D. 8 Ryan Ormond ${ }^{14}$, Sun Ha Paek ${ }^{15}$, Guido Reifenberger ${ }^{16}$, Peter A Sillevis Smitt ${ }^{13}$, Marion Smits ${ }^{17,18}$, Lucy $\mathrm{F}$ 9 Stead $^{19}$, Martin $\mathrm{J}$ van den Bent ${ }^{13}$, Erwin G Van Meir ${ }^{20}$, Annemiek Walenkamp ${ }^{21}$, Tobias Weiss ${ }^{22}$, Michael 10 Weller $^{22}$, Bart A Westerman ${ }^{23}$, Bauke Ylstra ${ }^{24}$, Pieter Wesseling ${ }^{18,24,25}$, Anna Lasorella ${ }^{26}$, Pim J French ${ }^{4}$, 11 Laila M Poisson ${ }^{2}$, The GLASS Consortium ${ }^{27}$, Roel GW Verhaak ${ }^{5,31}$, Antonio lavarone ${ }^{28,31}$, Houtan 12 Noushmehr ${ }^{2,31, *}$

\footnotetext{
1 School of Pharmaceutical Sciences of Ribeirão Preto, University of São Paulo, Ribeirão Preto, São Paulo 14040-900, Brazil

2 Hermelin Brain Tumor Center, Henry Ford Health System, Detroit, MI 48202, USA

3 Institute for Cancer Genetics, Columbia University Medical Center, New York, NY 10032, USA

4 Neurology Department, The Brain tumour center, Erasmus MC Cancer Institute, Rotterdam 3015 GD, the Netherlands

5 The Jackson Laboratory for Genomic Medicine, Farmington, CT 06032, USA

6 National Cancer Institute, Bethesda, MD 20892, USA

7 Center for Biomedical Image Computing and Analytics, University of Pennsylvania, Philadelphia, PA 19104, USA

8 Department of Pathology and Laboratory Medicine, Perelman School of Medicine, University of Pennsylvania, Philadelphia, PA 19104, USA

9 Department of Radiology, Perelman School of Medicine, University of Pennsylvania, Philadelphia, PA 19104, USA

10 Olivia Newton-John Cancer Research Institute, Austin Health, Heidelberg 3084, Melbourne, Australia

11 Luxembourg Institute of Health, Luxembourg L-1526, Luxembourg

12 Duke University, Durham, NC 27710, USA

13 Department of Neurology, Cancer Center Amsterdam, Amsterdam UMC, Vrije Universiteit Amsterdam, 1081 HV Amsterdam, the Netherlands

14 University of Colorado School of Medicine, Department of Neurosurgery, Aurora, CO 80045, USA

15 Seoul National University, Seoul, South Korea

16 Institute of Neuropathology, Heinrich Heine University, Moorenstrasse 5, D-40225 Duesseldorf, Germany

17 Department of Radiology and Nuclear Medicine, Erasmus MC, Rotterdam 3015 GD, the Netherlands

18 The Brain Tumour Centre, Erasmus MC Cancer Institute, Rotterdam, the Netherlands

19 Leeds Institute of Medical Research, University of Leeds, Leeds LS9 7TF, UK

20 Department of Neurosurgery and O'Neal Comprehensive Cancer Center, University of Alabama at Birmingham, Birmingham, AL 35233, USA

21 University of Groningen, Groningen, the Netherlands

22 Department of Neurology, University Hospital and University of Zurich, Frauenklinikstrasse 26, CH-8091 Zurich, Switzerland

23 Department of Neurosurgery, Brain Tumor Center, Cancer Center Amsterdam, Amsterdam UMC, Vrije Universiteit Amsterdam, 1081 HV Amsterdam, the Netherlands

24 Department of Pathology, Cancer Center Amsterdam, Amsterdam UMC, Vrije Universiteit Amsterdam, 1081 HV Amsterdam, the Netherlands

25 Princess Máxima Center for Pediatric Oncology, Utrecht, the Netherlands

26 Department of Pediatrics, Department of Pathology and Cell Biology, Institute for Cancer Genetics, Irving Comprehensive Cancer Center, Columbia University Medical Center, New York, NY 10032, USA

27 A list of participants and their affiliations appears in the online version of the paper

28 Department of Neurology, Department of Pathology and Cell Biology, Institute for Cancer Genetics, Irving Comprehensive Cancer Center, Columbia University Medical Center, New York, NY 10032, USA

29 Co-first author

30 Co-second author

31 Co-senior author

* Correspondence: houtan.noushmehr@hfhs.org
} 


\section{Summary}

15 Tumor adaptation or selection is thought to underlie therapy resistance of gliomas. To

16 investigate the longitudinal epigenetic evolution of gliomas in response to therapeutic

17 pressure, we performed an epigenomic analysis of 143 matched initial and recurrent

18 patients with IDH-wildtype (IDHwt) and IDH-mutant (IDHmut) gliomas. IDHwt gliomas

19 showed a longitudinally stable epigenome with relatively low levels of global

20 methylation, whereas the epigenome of IDHmut gliomas showed initial high levels

21 genome-wide of DNA methylation that was progressively reduced to levels similar to

22 those of IDHwt tumors. By integrating DNA methylation and gene expression data,

23 adaptive changes of putative master regulators of the cell cycle and of differentiation

24 were seen in IDHmut recurrent tumors. Furthermore, relapses of IDHmut tumors were

25 accompanied by histological progression which in turn influenced survival, as validated

26 in an independent cohort. Finally, the initial cell composition of the tumor

27 microenvironment differed between IDHwt and IDHmut tumors and changed

28 differentially following treatment, suggesting increased neo-angiogenesis and T-cell

29 infiltration upon treatment for IDHmut gliomas. Our study provides one of the largest

30 cohorts of paired glioma samples profiled with epigenomics, transcriptomics and

31 genomics; and our results demonstrate that the treatment of IDHmut gliomas reshapes

32 the epigenome towards an IDHwt-like phenotype. Accordingly, the prevalent practice of

33 early genotoxic treatment in this patient population may need to be revisited. 


\section{Introduction}

Despite advances in our biological understanding, molecular classification and surgical techniques, management of diffuse gliomas of adulthood remains challenging making it an incurable disease (Malta et al., 2018a). Compared to gliomas of the same grade that carry intact isocitrate dehydrogenase (IDH) 1 and 2 genes, gliomas with IDH

41 mutations exhibit a less aggressive clinical course which has led to their separation as

42 distinct tumor types in the 2016 World Health Organization (WHO) classification of

43 Tumors of the Central Nervous System (CNS) (Louis et al., 2016). Based on the revised

442021 WHO Classification (Louis et al., 2021), IDH-mutant tumors now comprise two

45 distinct tumor types, namely "oligodendroglioma, IDH-mutant and 1p/19q-codeleted

46 CNS WHO grade 2 or 3", and "astrocytoma, IDH-mutant CNS WHO grade 2, 3 or 4".

47 Yet, there is controversy on the morphological criteria used to distinguish CNS WHO

48 grades 2 and 3, and homozygous CDKN2A loss (a signature lesion of CNS WHO grade

494 among IDH-mutant astrocytomas), which is presently the only diagnostic molecular

50 marker in these tumors (Shirahata et al., 2018; Brat et al., 2020; Tesileanu et al., 2021).

51 Thus, additional molecular characterization is needed to establish which of these tumors

52 will rapidly progress or remain quiescent for several years with or without adequate

53 therapy (Weller et al., 2021). 
Epigenetics play a vital role in stratifying CNS tumors and gliomas into clinically

55 relevant subtypes (Capper et al., 2018; Ceccarelli et al., 2016). The Glioma-CpG Island

56 Methylator Phenotype (G-CIMP) discovered in IDH-mutant gliomas in 2010 (Noushmehr

57 et al., 2010) provided an explanation for their favorable prognosis. Further investigation

58 revealed a subset of IDH-mutant gliomas that presented with a lower degree of DNA

59 methylation and poorer outcome, named G-CIMP-low; distinct from the previously

60 described highly methylated tumors that have a better outcome, and renamed as G-

61 CIMP-high (Ceccarelli et al., 2016). In our previous study, longitudinal epigenomics

62 analyses of 77 paired glioma samples revealed that $12 \%$ of G-CIMP-high cases

63 progressed to G-CIMP-low cases (de Souza et al., 2018) highlighting the importance of

64 further studying epigenetic reprogramming following treatment and integrating it with

65 other genomic platforms to discover new prognostic markers and therapeutic targets.

66 Longitudinal analyses that integrate epigenetic and transcriptomic data offer an

67 opportunity to define key master regulators driving the malignant progression of each

68 glioma entity characterizing these prognostic subtypes as tumors transition to

69 recurrence after initial clinical management.

70 Current management and treatment for gliomas include surgery followed by

71 radiotherapy and/or alkylating chemotherapy (e.g. temozolomide [TMZ]). Recent studies

72 have revealed fundamental molecular genetic changes associated with glioma

73 treatment including the development of a hypermutation phenotype (Johnson et al.,

74 2014; Hunter et al., 2006), increase in small deletion burden and acquisition of CDKN2A

75 homozygous deletions associated with radiotherapy and acquired aneuploidy

76 associated with cell cycle related genes and overall poorer outcome (Barthel et al., 
77 2019; Kocakavuk et al., 2021). Interestingly, not all TMZ gliomas develop a

78 hypermutator status which challenges the possible mechanisms driving this TMZ

79 treatment-induced molecular phenotype (Mathur et al., 2020; Touat et al., 2020).

80 However, treatment-induced hypermutation related to epigenetic and transcriptomic

81 changes has not been well explored. It offers opportunities to identify potential

82 epigenetic markers for predicting transition to a hypermutator status.

In the current study, by leveraging the Glioma Longitudinal AnalySiS (GLASS)

84 international consortium (Barthel et al., 2019; GLASS Consortium, 2018; Varn et al.,

85 2021), we analyze an epigenetic cohort of 143 glioma patients with matched initial and

86 recurrent tumors, and include additional molecular data and clinical data to characterize

87 the evolution of both IDH-wildtype and IDH-mutant gliomas. This is the largest cohort of

88 paired initial and recurrent glioma samples profiled with epigenomics, transcriptomics

89 and genomics, that we know of being used in the literature. Also, the GLASS-NL

90 (GLASS in the Netherlands), a collaboration of several centers in the Netherlands

91 treating patients with glioma, was included in this study to evaluate the effects of

92 treatment in the epigenome of gliomas in an independent cohort. This consortium has

93 collected material from $100 \mathrm{IDH}$-mutant astrocytoma patients who underwent at least

94 two surgical resections (surgical interval $>6$ months). Our study aimed at identifying key

95 master regulators of tumor progression, identifying changes in the tumor

96 microenvironment and epigenetic drivers of glioma evasion to treatment and examining

97 differences in these processes between IDH-wildtype and IDH-mutant gliomas to derive

98 better informed tailored treatments. 


\section{Results}

100

101

102

103

104

105

106

107

108

109

110

111

112

113

114

115

116

117 subtypes (Codel, G-CIMP-high, and G-CIMP-low) and three IDHwt-specific subtypes

118 (Classic-like, Mesenchymal-like, LGm6) (Ceccarelli et al., 2016) (Figure 1B) and we

119 used this molecular classification throughout the analyses in this study. Over time, $25 \%$

$120(19 / 77)$ of IDHmut and 30\% (20/66) of IDHwt tumors switched subtypes. Among IDHmut

121 cases that switched subtypes, tumors of 13 patients $(13 / 19,68 \%)$ switched from G- 
122 CIMP-high to G-CIMP-low, a subtype associated with worse overall survival (time from

123 initial surgery to death or last follow-up). Among IDHwt cases that switched subtype,

124 tumors of 10 patients $(10 / 20,50 \%)$ switched from classic-like or LGm6 to the 125 mesenchymal-like subtype. Less frequent subtype shifts were also observed. A Pan126 CNS DNA methylation-based classification (Heidelberg subtype; Capper et al., 2018) 127 and a recent pathway-based classification of glioblastomas (Functional subtype; 128 Garofano et al., 2021) were also assigned to our cohort (Figure 1B). Based on the Pan129 CNS DNA methylation-based classification, 26 IDHmut astrocytomas (26/55, 47\%) 130 progressed to high-grade astrocytoma upon recurrence; whereas the IDHwt cases, 131 tumors of 7 patients $(7 / 22,32 \%)$ switched from mesenchymal to RTK II, 6 cases $(6 / 22$, 132 27\%) switched from RTK II to mesenchymal and $3(3 / 9,33 \%)$ from RTK I to 133 mesenchymal.

134 When the patients were stratified according to the genome-wide gain or loss of 135 DNA methylation groups upon recurrence, patients with IDHmut gliomas showed a 136 higher proportion of samples losing DNA methylation than patients with IDHwt gliomas 137 (42\% vs 6\%; Fisher's test, p < 0.0001; Figure 1B). Interestingly, 6 of 8 (75\%) of IDHmut 138 tumors that switched molecular subtypes and had genomic data available evidenced 139 hypermutator phenotype at recurrence (Figure 1C, Table S3B). Five of these switched 140 from G-CIMP-high to G-CIMP-low. The tumor of one patient switched from IDHmut 141 Codel to G-CIMP-high (non-codel subtype). In contrast, only 1 of 21 (5\%) IDHmut 142 patients which retained their subtype became hypermutator at recurrence (Fisher's test, $143 \mathrm{p}=0.0004)$. All switches were toward a more aggressive phenotype (e.g. G-CIMP-low 
144 and grade 4), suggesting an association between DNA methylation change, tumor 145 progression, and hypermutation acquisition.

To further explore the association of epigenomic changes and hypermutation, we

147 investigated the methylome of the initial tumors of patients that subsequently received 148 chemotherapy, particularly TMZ, to identify biomarkers that could predict hypermutation 149 in gliomas. We identified 6 IDHmut and 4 IDHwt initial tumors that transformed to 150 hypermutators upon the first recurrence and had treatment information available (Figure 151 S1A). For comparison, we identified 5 IDHmut and 5 IDHwt samples that, despite 152 receiving $\mathrm{TMZ}$ and/or $\mathrm{RT}$ after initial surgery, did not become hypermutators which were 153 used as controls.

154 A supervised analysis between initial IDHmut gliomas that did or did not develop 155 a hypermutator phenotype at recurrence resulted in 342 differentially methylated CpG 156 probes (unadjusted $p$-value $<0.01$ and DNA methylation difference $>25 \%$ ) (Figure S1B, 157 Table S5). To understand the biological implications of these DNA methylation changes, 158 we integrated the methylome data with RNA-sequencing and selected CpGs within the 159 promoter region of genes and identified 23 CpG-gene pairs inversely correlated, such 160 as the DNA replication initiator gene REPIN1 and the gene $K C N K 2$, which has been 161 associated with positive regulation of cellular response to hypoxia (Wu et al. 2013), 162 indicating potential mechanisms associated with the predisposition to hypermutation 163 (Figure S1C). Within IDHwt tumors, we identified 548 CpG probes that were associated 164 with hypermutation in IDHwt gliomas (unadjusted p-value $<0.05$ and DNA methylation 165 difference $>30 \%$ ) and 14 genes being negatively correlated with CpG methylation, 166 including MGMT and the gene NETO1, which is associated with regulation of long-term 
neuronal synaptic plasticity ( $\mathrm{Ng}$ et al. 2009) (Figure S1D and S1E, Table S5). Further studies would be needed to confirm the predictive power of these signatures.

Despite evidence that hypermutation might occur in random chromosomal 170 locations (Touat et al., 2020), we investigated the methylome of the recurrent 171 hypermutated tumors to identify regions with consistent patterns of DNA methylation.

172 We compared the epigenome between first recurrence hypermutators ( $\mathrm{N}=8$ IDHmut; 7 173 IDHwt) and non-hypermutators ( $\mathrm{N}=11$ IDHmut; 20 IDHwt), stratified by IDH status. In 174 the IDHmut group, we identified 502 differentially methylated CpG probes (DMP) 175 (unadjusted $p$-value $<0.01$ and DNA methylation difference $>30 \%$ ) that defined the 176 hypermutator phenotype in gliomas, the majority of which were hypomethylated (Figure 177 S1F, Table S5). In IDHwt tumors, the set of CpG probes identified by our analysis $178(\mathrm{~N}=620 ;$ unadjusted $\mathrm{p}$-value $<0.05$ and DNA methylation difference $>40 \%)$ showed that 179 the hypermutators are mostly hypermethylated compared to non-hypermutators 180 ( $\mathrm{N}=94 \% ; 581 / 620)$ (Figure S1G, Table S5). Taken together, these findings suggest that 181 epigenetic biomarkers predict the predisposition to hypermutation in patients who 182 receive $\mathrm{TMZ}$ after the initial surgery. Further validation studies could help elucidate the 183 biological role of these regions in determining the susceptibility to hypermutation.

To further investigate the changes in the epigenome that occur over time in gliomas, we compared the genome-wide DNA methylation characteristics of the initial 187 compared to recurrent tumor samples, stratified by IDH status (Figure S2B). IDHwt 188 gliomas showed a more stable epigenome over time (i.e. zero CpG probes presented a 189 differentially methylated mean difference greater than $20 \%$ ), while the epigenome of 
190 IDHmut gliomas showed genome-wide loss of DNA methylation (593 CpG probes with

191 DNA methylation difference > 20\%) throughout the disease evolution (Figure S2B).

192 IDHmut patients that progressed from G-CIMP-high to G-CIMP-low showed the most

193 prominent loss of DNA methylation, particularly at intergenic regions, which suggests an

194 association between the loss of DNA methylation and tumor progression in this subtype

195 (Figures S2C, S2D and S2E), confirming previous results from our group and others

196 (Nomura et al., 2019; de Souza et al., 2018). Compared to patients with tumors that

197 remained G-CIMP-high at recurrence, those with recurrent G-CIMP-low more often had

198 histologically higher-grade astrocytoma, were less often managed by a watch-and-wait 199 strategy and exhibited inferior survival (Table S3A).

200 Next, we investigated the impact of the epigenomic changes on transcriptional 201 networks using an integrative approach that combines epigenome and transcriptome to 202 define master regulators. We applied ELMER (Enhancer Linking by 203 Methylation/Expression Relationships) (Silva et al., 2019; Yao et al., 2015), a 204 computational tool that harnesses DNA methylation to identify regulatory elements and 205 correlates the enhancer state with the expression of nearby genes to define putative 206 transcriptional targets. ELMER also allows the inference of transcription factor (TF) 207 binding motif analysis and the integration of TF expression to identify master regulatory 208 TFs (MRTFs) (Silva et al., 2019).

We compared initial and recurrent IDHmut gliomas and identified a total of 1,590 210 DMP between the groups (FDR < 0.05, Figure 2A, S4A). To better understand the 211 genomic context of the DNA methylation changes associated with the progression of 212 IDHmut gliomas, we profiled nine IDHmut G-CIMP-high gliomas at diagnosis and three 
213 recurrences evolving into IDHmut G-CIMP-low states with ChIP-seq for the H3K27Ac

214 active chromatin mark. The integration of DMP with the local changes of H3K27Ac (20

215 nearest genes of each $\mathrm{CpG}$ probe in the methylation array) converged on the

216 identification of 1,282 genes exhibiting coordinated loss of DNA methylation and gain of

$217 \mathrm{H} 3 \mathrm{~K} 27 \mathrm{Ac}$, thus indicating that this gene set is epigenetically activated by loss of DNA

218 methylation at recurrence. A pathway-based analysis of these genes suggested 219 enrichment of cell cycle and proliferation-related activities (Figure 2B, Table S4B).

220 Within the regulatory DMP regions, we identified the enrichment of 32 distal221 binding motifs for specific TFs with TGAGTCA (Fos/Jun family) and the enhancer-box 222 CAGATGG being the most represented (Figure 2A, Table S4C). By computing the 223 anticorrelation of TF gene expression and the DNA methylation levels within their 224 binding sites, we identified candidate TFs with increasing expression in IDHmut 225 recurrent tumors to levels comparable to those of IDHwt gliomas (Figure 2C). Some of 226 these master regulatory (MR) TFs are well-established glioma oncogenes: FOS/JUN 227 family members have been associated with control of tumor malignancy (Blau et al., 228 2012), cell proliferation and radioresistance (Liu et al., 2016), and glioma progression 229 (de Souza et al., 2018); musculin (MSC) has been reported to inhibit differentiation of 230 embryonal carcinoma (Yu et al., 2003) and B-cell lymphoma cells (Mathas et al., 2006). 231 Indeed, recurrent IDHmut tumors show higher stemness activity (as defined by our 232 recent study) (Malta et al., 2018b) than the corresponding initial tumors (Figure 2D). We also identified 201 DMP between initial and recurrent IDHwt glioma samples,

234 which were mapped to both distal regions (156 CpG probes) and promoters (45 CpG 235 probes) and their putative associated genes (Figure S2F and S2G). We did not identify 
236 any TF binding motifs enriched within the DMP regions, showing again less frequent

237 changes in epigenetic regulation in IDHwt tumors over time.

238 Together, our results show that IDHmut gliomas present a more dynamic

239 epigenome, characterized by loss of DNA methylation at recurrence, with activation of

240 oncogenes to expression levels similar to those observed in IDHwt gliomas. In contrast,

241 the epigenome of IDHwt gliomas seems relatively preserved longitudinally. We

242 identified potential master regulators driving cell cycle deregulation in IDHmut gliomas

243 at recurrence, which represent potential treatment targets.

244 DNA methylation loss associated with recurrent IDHmut gliomas after standard 245 treatment

246 It has been shown that treatment (radiotherapy and alkylating chemotherapy) improves

247 the progression-free survival of IDHmut gliomas (Wick et al., 2009). However,

248 recurrence of IDHmut lower-grade glioma is frequently associated with progression to 249 higher histological grades. Treatment of IDHmut lower-grade gliomas with TMZ and/or

250 radiotherapy has been linked to many genomic alterations, such as a hypermutator

251 phenotype (Johnson et al., 2014; Touat et al., 2020), and a strong tendency towards

252 aneuploidy and a specific radiotherapy-associated deletion signature by genetic

253 analysis (Kocakavuk et al., 2021). Herein, we observe that treatment is associated with

254 epigenomic changes. Whether and how to treat a low-grade IDH-mutant glioma is still

255 not based on high level evidence from controlled clinical trials; we sought to identify the

256 DNA methylation changes triggered at recurrence by the different treatment choices

257 made in our cohort. Towards this goal, we divided our cohort into 4 groups: patients

258 who received $T M Z$ only $(N=12)$, radiotherapy $(R T)$ only $(N=18)$, the combination of $R T$ 
and $\mathrm{TMZ}(\mathrm{RT}+\mathrm{TMZ} ; \mathrm{N}=6)$ and patients who did not receive additional treatment after the

260 first surgery but were managed by a watch-and-wait approach ( $N=33$ ) (Tables S3D and

261 S6). The supervised methylome analysis of first recurrent IDHmut gliomas across the 4

262 groups defined 620 DMP (Kruskal-Wallis test by ranks, FDR $<0.01$ and DNA

263 methylation difference $>$ 20\%) (Figure 3A, Table S6). Upon first investigation, we

264 determined that these CpGs were associated with consistent loss of DNA methylation in

265 patients who received any treatment besides surgery after initial diagnosis (TMZ only,

266 RT only or combined TMZ and RT) compared to their primary counterparts. On the

267 other hand, the methylome of the recurrent sample of patients who did not receive

268 treatment resembled the initial tumor (Figure 3A). There were no CpG probes that

269 distinguished the different groups that received any specific type of treatment (TMZ

270 only, RT only or $\mathrm{RT}+\mathrm{TMZ}$ ), suggesting that the introduction of any treatment regimen

271 involving TMZ and/or RT after initial surgery is sufficient to remodel the methylome of

272 treatment-naïve gliomas. The interrogation of the same CpGs corroborated this

273 observation in IDHwt initial and recurrent gliomas, which showed a similar DNA

274 hypomethylation profile to the IDHmut treatment arm (TMZ only, RT only and RT+TMZ)

275 (Figure 3A). To evaluate whether the observed decrease of DNA methylation in our

276 discovery cohort (GLASS-International; this study) is consistent in an independent

277 cohort, we sought to validate our findings in a yet unpublished dataset of paired glioma

278 samples from the GLASS in the Netherlands consortium (GLASS-NL; validation)

279 (GLASS Consortium, 2018). The validation cohort consists of 36 treated paired glioma

280 samples and 64 untreated paired samples, all of which are IDHmut astrocytomas at

281 diagnosis. The loss of DNA methylation pattern upon treatment after initial surgery was 
282 confirmed in the validation cohort (Figures 3B and 3C). Because the GLASS-NL cohort

283 was comprised of only IDHmut astrocytomas, we repeated our discovery test on only

284 the IDHmut astrocytomas of the GLASS-International cohort (excluding

285 oligodendroglioma samples), separated by treatment group and selected a new set of

286 DNA hypomethylated $\mathrm{CpG}$ probes $(\mathrm{N}=981)$ in the treated samples (FDR $<0.01$ and

287 DNA methylation difference > 25\%). 61\% (381/620) of the previously described CpG

288 probe list overlapped with this new signature showing consistent DNA methylation

289 changes. Again, the loss of DNA methylation pattern after treatment was confirmed in

290 the validation cohort (Figure S3A, Table S6).

291 By comparing different clinical and molecular features of these tumors, we

292 observed a significant enrichment of progression to IDHmut astrocytoma CNS WHO 293 grade 4 in the treatment vs the non-treatment groups in the discovery (Fisher's test = 294 0.03; Figure 3D) but not with the validation datasets (Fisher's test $p=0.3$; Figure S3B).

295 In addition, we noticed that 34\% (10/29) of G-CIMP-high tumors progressed to G-CIMP-

296 low in the treatment group vs $4 \%(1 / 27)$ in the non-treatment group (Fisher's test, $297 \mathrm{p}=0.005)$. The same was observed with the molecular Pan-CNS classification (Capper 298 et al., 2018), in which 68\% (15/22) of IDHmut astrocytomas progressed to high-grade 299 astrocytoma in the treatment group vs $23 \%(6 / 26)$ in the non-treatment group (Fisher's 300 test, $p=0.003$, Table S1). As expected, only in the treatment group, we observed 301 hypermutator samples $(\mathrm{N}=5)$ (Figure $3 \mathrm{~A})$. To consider whether the selection of the 302 treatment regimen might have been biased by the age at diagnosis, we performed an 303 analysis of variance (ANOVA) on age at the initial tumor resection and we did not find 304 an age difference across the four groups (ANOVA F-test $p$-value $=0.59$ ). To explore the 
305 biological implications of these epigenomic changes, we searched for inversely

306 correlated DNA methylation and gene expression. We identified 24 distal CpG-gene 307 pairs $(F D R<0.01)$ enriched for the NEUROD1 motif resulting in 18 unique genes 308 potentially regulated by DNA methylation after treatment (Figures 3E and S3C, Table 309 S6) which included known oncogenes and cancer-related genes such as MYB and 310 MYO15A and enriched for monocarboxylic acid metabolic process.

311 Tumor microenvironment changes and clinical implications of treatment in IDH-mutant 312 gliomas revealed by DNA methylation

As the observed changes of DNA methylation may not only be driven by tumor

314 cell-intrinsic-events but may also underscore changes in the methylome associated with

315 non-tumor cell populations present in the glioma tumor microenvironment (TME), we 316 applied a methylation-specific approach for the deconvolution of non-tumor cells to the 317 longitudinal glioma cohort. MethyICIBERSORT (Chakravarthy et al., 2018) uses 318 genome-wide DNA methylation data to deconvolute cell types from individual tumor 319 samples. Our estimated cell populations consisted of 10 cell types: glia, neuron, 320 endothelial cells (CD31+), B cells (CD19+), CD4 effector T cells, CD8+ T cells, $T$ regs, 321 natural killer cells (CD56+), macrophages and neutrophils. Compared to untreated 322 recurrencies, recurrent IDHmut tumors which were treated after surgery were marked 323 by increased infiltration of endothelial cells (CD31+) and CD8 T lymphocytes (CD8+)

324 (Figure 4A, Table S7), suggesting that treatment impacts the tumor microenvironment 325 and most notably angiogenesis. Our in silico cell fraction estimation was validated by 326 immunohistochemical staining in representative IDHmut samples that received 327 treatment after initial surgery (Figure 4B). As a further validation, the relative proportion 
of non-tumor cells estimated by methyICIBERSORT was significantly correlated with the

329 glioma cell compartments estimated by gene expression for the GLASS-international

330 transcriptomic cohort (Varn et al., 2021) (Figure S4A). Additional changes in the TME

331 were also observed within IDHmut and IDHwt longitudinally, particularly a significant

332 increase in macrophage in recurrent IDHwt tumors (Figures S4B-S4D).

333 To evaluate the clinical implications of these findings, we next assessed the

334 clinical follow-up of the entire IDHmut GLASS-International cohort divided into treatment

$335(\mathrm{~N}=73)$ or non-treatment $(\mathrm{N}=37)$ groups. First, patients receiving treatment after initial

336 surgery had a worse survival than patients who did not receive treatment beyond

337 surgery (log-rank p-value=0.03, Figure 4C, left). However, patients left untreated after

338 initial surgery had a progression-free interval (PFI) of 27 months that was significantly

339 worse compared to a PFI of 40.5 months in the treatment group (log-rank $p=0.009$,

340 Figure 4C, middle), thus confirming the results of EORTC 22845 (van den Bent et al.,

341 2005). When we focused on the survival interval from second surgery to the last follow-

342 up, as expected, we found a markedly worse survival in previously treated patients (log-

343 rank $p=0.0001$, Figure $4 \mathrm{C}$, right). The same associations of treatment and clinical

344 implications were observed in the validation GLASS-NL cohort (Figure 4D).

\section{Discussion}

This work reports and analyzes the largest cohort of matched glioma samples 348 profiled with epigenomics, transcriptomics, and genomics platforms to uncover the

349 diverse molecular routes that drive treatment-resistant gliomas during progression. By 
350 applying an integrative molecular approach, we highlight the critical epigenetic

351 mechanisms by which gliomas evaded treatment in addition to transcriptomic and

352 genetic evolution. We also identified epigenomic changes that may be useful as

353 biomarkers for continuous monitoring of disease progression and treatment response

354 prediction.

355 Our cohort consisted of the three major glioma subtypes (IDHmut-noncodel, 356 IDHmut-codel and IDHwt) and revealed key evolutionary differences across these 357 subgroups. Earlier work from us and others described the G-CIMP glioma phenotype in 358 IDHmut glioma characterized by higher levels of DNA methylation likely as a direct 359 result of the epigenetic effects of the oncometabolite 2-hydroxyglutarate that is 360 generated by mutant IDH enzymes (Noushmehr et al., 2010; Turcan et al., 2012). 361 These tumors exhibited favorable clinical outcomes compared with IDHwt gliomas, 362 which have lower DNA methylation levels (Noushmehr et al., 2010). Indeed, in our 363 previous work, we reported that the extent of genome-wide DNA methylation showed 364 broad positive correlation with clinical outcome for IDHwt glioma, now defined as 365 "molecular GBM" given their aggressive behavior independent of histological grading 366 (Louis et al., 2001), with these tumors having the lowest levels of DNA methylation 367 genome-wide. Here we confirm and extend these notions to tumor progression. In 368 particular, we found that IDHwt gliomas presented an initial low genome-wide DNA 369 methylation, which shows minimal changes during the course of the disease. This is 370 consistent with the malignant state of IDHwt gliomas at diagnosis and which does not 371 appear to progress during treatment. 
Conversely, we uncovered pronounced epigenetic changes in IDHmut gliomas,

373 which invariably converged toward lower DNA methylation levels in recurrent treated

374 tumors compared to untreated neoplasms. In the most extreme cases, the treatment-

375 induced evolution of IDHmut glioma resulted in a state of DNA hypomethylation

376 comparable to IDHwt gliomas. Thus, the epigenetic trajectory of glioma progression was

377 associated with progressively lower levels of DNA methylation in IDHmut tumors or was

378 essentially moot in the case of IDHwt gliomas as glioma initiation in the absence of IDH

379 mutations coincided with the lowest possible levels of DNA methylation.

380 Following diagnosis and surgery, IDHmut glioma patients may or may not

381 undergo treatment with radiation or alkylating chemotherapy or both (Baumert et al.,

382 2016; Buckner et al., 2016). A recognized effect of alkylating agents typically used in

383 glioma therapy (temozolomide) is the potential acquisition of a hypermutator phenotype

384 (Barthel et al., 2019; Johnson et al., 2014). Here, we found that treatment with radiation

385 and/or chemotherapy individually or combined accelerated progression towards the

386 hypomethylated state at recurrence. These findings were confirmed in an independent

387 cohort, suggesting that current treatment regimens alter the epigenomic landscape of

388 IDHmut gliomas similar to the observed treatment-induced genomic abnormalities in

389 response to alkylating agents.

390 The treatment-accelerated epigenetic drift towards the hypomethylated state

391 parallels the histopathological shift from a lower- to a higher-grade phenotype.

392 Altogether, these results indicate that the initial treatment with radiotherapy and/or

393 temozolomide may have triggered a more aggressive evolution at the time of the tumor

394 recurrence which compromised the survival probability of these patients at that 
recurrence. More specifically, the introduction of treatment after initial surgery in IDH-

396 mutant gliomas is associated with a significant delay in tumor progression. The time

397 from second surgery to progression or death is significantly shorter when compared to

398 untreated patients which is likely associated with the loss of DNA methylation and

399 activation of associated genes that we uncovered. While these findings remain

400 consistent with previous observations from large clinical trials that reported the 401 beneficial role of chemotherapy and radiotherapy for patients survival (van den Bent et

402 al., 2005), they also highlight the ability of radio/chemotherapy to trigger a markedly 403 more aggressive behavior at recurrence and identify the epigenetic determinants of the 404 treatment-induced glioma progression.

405 The therapy-associated changes of the epigenetic evolution of IDHmut glioma 406 were also mirrored by specific changes in the tumor microenvironment (TME) at 407 recurrence. At recurrence, CD8 and endothelial cell-related signatures were elevated in 408 treated IDHmut gliomas compared to untreated tumors. Together, these findings 409 indicate that the epigenomic and genomic changes associated with more aggressive 410 histotypes of IDHmut gliomas at recurrence coincided with specific changes of the TME 411 (neo-angiogenesis and changes in T-cell composition), indicating the convergence of 412 IDHmut glioma evolution towards features more typical to the most aggressive IDHwt 413 subtype.

414 Having established the importance of epigenetic evolution of IDHmut glioma, we 415 focused on this subtype to integrate the analysis of epigenome, transcriptome and 416 ChIP-seq to extract the features associated with the evolution towards loss of DNA 417 methylation and consequent transcriptional activation of critical drivers of progression 
418 (master regulators, MRs). First, the integration of DNA methylation and transcriptomic

419 platforms uncovered the convergence towards gene sets enriched with cell cycle and

420 proliferation-related activities and stemness, in comparison to initial tumors. Thus, such

421 biological activities are directly activated by transcriptional derepression associated with

422 local loss of DNA methylation. Second, we focused our attention on identifying MRs that

423 enabled the biological evolution of IDHmut glioma. MR are TFs regulating multiple

424 genes and in the case of cancer, drive the onset or progression of the disease.

Our approach depends on our understanding that discrete regions of the genome

426 harbors DNA sequence elements recognized by TFs. The presence of the TF and the

427 accessibility of the functional elements can then drive the activity of nearby or distant

428 genes. We uncovered a set of established oncogenes as MRs activated by

429 hypomethylation-induced derepression in IDHmut glioma, among them FOS/JUN family

430 members. Interestingly, therapeutic targeting of cJun transcription has included small

431 molecules interfering with its DNA binding domain, thereby preventing it from activating

432 downstream target genes such as those represented in our study. Two such molecules

433 with potential therapeutic advantages are MLN944 (Dai et al., 2004) and retinoid

434 (SR11302) which could be explored in a follow-up study involving recurrent glioma

435 tumors.

$436 \quad$ In summary, we found that upon tumor progression after standard treatment loss

437 of DNA methylation in IDHmut tumors is frequent which results in epigenetic activation

438 of cell cycle-related genes associated with early tumor progression and alterations in

439 the TME contexture towards angiogenesis and a T-cell composition that resembles a

440 treatment-naive IDHwt glioma (Figure 5). In untreated IDHmut patients the epigenome 
does not change significantly, and tumors progress later than the treated ones, either spontaneously or after subsequent treatment.

\section{Figure Legends:}

Figure 1. Epigenomic evolution of matched initial and recurrent gliomas. A) Venn diagram of number of patients who had DNA methylation, genomic (WGS/WXS) and/or RNAseq profiling. B) Clinical and molecular overview of matched initial and recurrent DNA methylation cohort. Each column represents a single patient $(n=143)$ at two separate time points grouped by IDH status and ordered by increase of gain of DNA methylation at recurrent tumor from left-toright. Top plot shows the surgical interval of each patient. C) Frequency of patients with hypermutator tumors that switched or retained molecular subtype at recurrence. Patients are divided by IDH status.

Figure 2. Master regulators associated with IDHmut glioma recurrence/progression. A) The outer track shows chromosome location. The second track shows genomic location of 1,590 differentially methylated CpG probes between initial and recurrent IDHmut tumors. Height of dots represents the difference of methylation levels between recurrent and initial. The third track shows the genomic location of $\mathrm{H} 3 \mathrm{H} 27$ ac peaks defined by differential binding analysis of IDHmut G-CIMP-high patients that progressed to G-CIMP-low. Height of the peaks represents the fold change of differential binding analysis. The fourth inner track shows the genomic location of the candidate genes epigenetically regulated by the CpG probes (yellow bars). Height of the bars represents the -log of FDR. Genes related to cell cycle are labeled. The top transcription factor (TF) binding motifs enriched among the candidate DMP are FOS/JUN family and NEUROD1, whose DNA signature motifs are represented in the center of the circos plot. B) Pathway analysis of epigenetically activated genes in recurrent IDHmut tumors. C) Gene expression levels of master regulator transcription factors (MRTFs) of IDHmut initial and recurrent tumors. IDHwt tumors are shown as a reference. D) Stemness activity in glioma samples stratified by primary/recurrent and IDH mutation status. analysis of $620 \mathrm{CpG}$ probes that are associated with different treatment strategies in IDHmutant paired glioma samples. Columns represent glioma samples, rows represent $\mathrm{CpG}$ probes. Samples were stratified and clustered based on IDH mutation status and primary/recurrent status and CpGs were ordered using hierarchical clustering methods. Nonneoplastic brain samples are represented on the left of the heatmap. DNA methylation beta- 
values range from 0 (low) to 1 (high). Additional tracks are included at the top of the heatmaps to identify each sample membership within separate cluster analysis. B) Heatmap of DNA methylation data in the validation cohort - GLASS-NL, showing the same $620 \mathrm{CpG}$ probes of panel A. C) Boxplot of the average DNA methylation beta-value of the $620 \mathrm{CpG}$ probes from panel A. Samples are stratified by primary/recurrent status and by treated/non-treated status. Left: GLASS-International samples. Right: GLASS-NL samples. D) Evolution of tumor histology (2021 WHO classification) from primary to recurrent samples after treatment compared to nontreated gliomas. E) Scatter plot of mean DNA methylation of $\mathrm{CpG}$ probes and mean gene expression of the epigenetically regulated genes after treatment.

Figure 4. Tumor microenvironment and clinical implications of treatment in IDH-mutant gliomas. A) CD31 and CD8 proportions (range scaled from 0 to 100\%) in samples originating from IDHmut matched initial and recurrent tumors in treated and non-treated patients. B) Illustrative immunohistochemical stainings for two marker proteins (CD31 and CD8) in an individual patient showing change of levels of tumor-infiltrating immune cells between initial and recurrent tumors. C/D) Overall survival and surgical interval analysis of IDHmut gliomas for the GLASS International (C) and GLASS-NL (D) cohorts.

Figure 5. Schematic of treatment-induced epigenetic activation of cell cycle in IDHmut further epigenetic activation of cell cycle related genes associated with early tumor progression. In IDHmut non-treated patients, epigenetic changes are much more limited and tumors may progress later, either spontaneously or after subsequent treatment.

\section{Supplemental Figure Legends:}

Figure S1: DNA methylation signature to predict the hypermutator phenotype upon initial surgery divided by IDH status, related to Figure 1. A) Venn diagram of the GLASS hypermethylator cohort based on data type: Whole-genome sequencing, DNA methylation array and clinical follow-up. B) Heatmap of DNA methylation data across IDHmut samples at initial diagnosis that were treated with $T M Z$, stratified by hypermutator status. Supervised hierarchical clustering analysis of $342 \mathrm{CpG}$ probes that can predict the hypermutator status at the initial surgery. Column-wise represents 11 glioma samples, row-wise represents CpG probes. Samples and CpGs are ordered using hierarchical clustering method. DNA methylation betavalues range from 0 (low) to 1 (high). Additional tracks are included at the top of the heatmaps to identify each sample membership within separate cluster analysis. C) Boxplot of the CpGgene pairs at gene promoter regions of IDHmut gliomas divided by DNA methylation (top) and gene expression (bottom) data. D) DNA methylation heatmap of $548 \mathrm{CpG}$ data across IDHwt samples at initial diagnosis treated with $T M Z$, stratified by hypermutator status. E) Boxplot of CpG-gene pairs at gene promoter regions identified in IDHwt gliomas. F/G) Differentially methylated regions between hypermutator vs non-hypermutator recurrent samples divided by 
Figure S2. Longitudinal epigenomic changes of matched initial and recurrent gliomas, related to Figure 2. A) Venn diagram of DNA methylation samples which were profiled with genomics (WGS/WXS) and/or RNAseq. B) Overall genome-wide DNA methylation correlation between initial and recurrent tumors, stratified by IDH mutation status. C/D/E) Overall DNA methylation correlation between initial and recurrent tumors of different glioma subtypes, by CpG probe genomic location: $C$, genome-wide; D, probes located in intergenic regions, and $E$, CpG probes located in promoters of genes. F/G) Differentially methylated CpG probes defined between recurrent and initial IDHwt tumors and their associated putative genes. F, CpG probes located at distal regions and G, CpG probes located at promoter regions.

Figure S3. DNA methylation loss is associated with malignant progression of glioma after standard treatment in the validation cohort: GLASS-NL, related to Figure 3. A) Heatmap of DNA methylation data. Supervised analysis using astrocytoma-only samples from GLASSInternational cohort identified $981 \mathrm{CpG}$ probes that are associated with treatment astrocytomas IDHmut paired glioma samples. Samples from the validation cohort are also shown. Samples are stratified by cohort, initial/recurrent status and treatment status. Column-wise represents glioma samples, row-wise represents $\mathrm{CpG}$ probes. DNA methylation beta-values range from 0 (low) to 1 (high). Additional tracks are included at the top of the heatmaps to identify each sample membership within separate cluster analysis. B) Evolution of tumor histology (2021 WHO classification) of the validation cohort (GLASS-NL) after treatment compared to nontreated gliomas. C) Scatter plot of 24 CpG-gene pairs epigenetically regulated genes after treatment in IDHmut gliomas.

Figure S4. Glioma subtypes present different tumor microenvironments and it changes overtime, related to Figure 4. A) Correlation between cell composition estimated by DNA methylation and by gene expression. B) Barplots of the estimated median infiltration of specific cell types as a proportion of all cell types (range scaled from 0 to 100\%) in 143 glioma tumors at initial diagnosis, divided by IDH mutation status. C) Cell type proportion (range scaled from 0 to $100 \%$ ) in samples originating from the matched initial and recurrent tumors, divided by molecular subtypes. All comparisons (initial vs. recurrence, by subtype, for the specified cell population) are statistically significant $(P<0.05)$. $P$ values calculated using a two-sided Wilcoxon rank-sum test. Matched primary and recurrent tumors are linked by the lines. D) Representative immunohistochemical staining for CD163 marker protein in two individual patients with changing levels of tumor-infiltrating immune cells between initial and recurrent 


\section{Methods}

CONTACT FOR REAGENT AND RESOURCE SHARING

564 Further information and requests for resources and reagents should be directed to and will be fulfilled by the Lead Contact Houtan Noushmehr (hnoushm1@hfhs.org).

\section{Biospecimens/GLASS Datasets}

568 Datasets added to GLASS came from both published and unpublished sources. The 569 GLASS epigenomic cohort consists of 357 DNA methylation samples (143 patients) 570 profiled by either Illumina $450 \mathrm{~K}$ or EPIC Beadchip methylation arrays and described 571 below. For those same patients, we also collected DNA sequencing data of 157 572 genomic samples, WXS or WGS (69 patients); and RNA sequencing of 120 samples 573 (59 patients), available through the GLASS consortium, resulting in the largest cohort of 574 matched glioma samples profiled with epigenomics, transcriptomics and genomics 575 platforms.

576 Newly generated DNA methylation data was collected from four different institutions: 577 Henry Ford Hospital $(n=103)$, University of Leeds (UK) $(n=8)$, Chinese University of 578 Hong Kong $(n=6)$, and Luxembourg Institute of Health $(n=54)$. The DNA was extracted 579 at each institution. New DNA methylation data from Henry Ford Hospital and from 580 Chinese University of Hong Kong was generated at the University of Southern 581 California. Briefly, the DNA was bisulfite-converted (Zymo EZ DNA methylation Kit; 582 Zymo Research) and profiled using an Illumina Human EPIC array (EPIC). For the 583 Luxembourg Institute of Health samples, DNA methylation data was generated by the 584 Illumina EPIC array at the Helmholtz Zentrum München (Research Unit of Molecular 585 Epidemiology, Institute of Epidemiology, German Research Center for Environmental 586 Health, Neuherberg, Germany) or by the Laboratoire National de Santé 587 (Neuropathology Unit, National Center of Pathology, Dudelange, Luxembourg). 588 Samples from University of Leeds were profiled locally using Illumina 450K Beadchip 
589 methylation arrays. The raw DNA methylation intensity data files (IDAT) were processed 590 with the minfi package (Fortin et al., 2017). We performed noob (Normal-exponential 591 convolution using out-of-band probes) background correction (Triche et al., 2013) and 592 dye bias correction using the minfi package (v 1.36.0) (Fortin et al., 2017). The DNA 593 methylation value for each locus is presented as a beta $(\beta)$ value $(\beta=(M /(M+U))$ in 594 which $M$ and $U$ indicate the mean methylated and unmethylated signal intensities for 595 each locus, respectively. $\beta$-values range from zero to one, with scores of zero indicating 596 no DNA methylation and scores of one indicating complete DNA methylation. A 597 detection p-value also accompanies each data point and compares the signal intensity 598 difference between the analytical probes and a set of negative control probes on the 599 array. Any data point with a corresponding p-value greater than $1 \mathrm{E}-4$ is deemed not to 600 be statistically significantly different from background and was thus masked as "NA". All 601 data files that were used in our analysis can be found at 602 https://www.synapse.org/\#!Synapse:syn17038081/wiki/585622.

603 RNA expression data used in this study was downloaded from the GLASS portal and 604 the TPM data matrix was filtered for selected protein coding genes only. Next, batch 605 effects due to the different Aliquot Batches were corrected using the COMBAT algorithm 606 with aliquots as covariates (Leek et al., 2012).

\section{Public Datasets}

608 Public samples included in the GLASS cohort were downloaded from: TCGA/GDC 609 (https://portal.gdc.cancer.gov), (Bai et al., 2016; Mazor et al., 2015, 2017; de Souza et 610 al., 2018). The raw DNA methylation IDAT files were accessed and processed as 611 described for the GLASS datasets above. Sample IDs and tissue source sites from our 612 entire longitudinal glioma cohort are listed in Table S1.

\section{Quality control}

614 DNA methylation quality control was performed using the entire GLASS epigenetic 615 samples to ensure the identity check of samples matched to their corresponding patient. 616 The DNA methylation signals of probes querying high-frequency SNPs were used to 617 calculate a pairwise agreement score across samples (Heiss and Just, 2018). Only 
618 samples that passed our pairwise agreement score cutoff were kept in the GLASS 619 epigenetic cohort $(n=357)$.

\section{Classification of longitudinal gliomas}

621 Longitudinal glioma samples were classified as either IDH-wildtype (Classic-like, 622 Mesenchymal-like, LGm6) or IDH-mutant (Codel, G-CIMP-high, and G-CIMP-low) DNA 623 methylation subtypes using the CpG methylation signatures and method previously 624 defined by our group (Ceccarelli et al., 2016).

625 Our cohort was also classified into the Pan-CNS DNA methylation-based classification 626 (Capper et al., 2018) by uploading idat files into the portal 627 https://www.molecularneuropathology.org/mnp.

628 Additionally, the samples were classified into the recent transcriptomic pathway-based 629 classification of glioblastomas (Garofano et al., 2021) using MWW-GST on the basis of 630 the highest positive Normalized Enrichment Score (Frattini et al. 2018).

631 Hypermutation was defined for all recurrent tumors that had received TMZ after initial 632 surgery and had more than 10 mutations per megabase sequenced, as described 633 previously (Barthel et al., 2019).

\section{Identification of master regulator transcription factors}

635 Initial and recurrent glioma samples were compared using the package ELMER (Silva et 636 al., 2019) for the following pair-wise comparison: IDHmut initial vs recurrent (20 637 matched pairs), IDHwt initial vs recurrent (29 pairs), and IDHmut treated vs untreated 638 (20 matched pairs). Only samples that have both DNA methylation and gene expression 639 available were used in these analyses.

640 We applied the default probe filter "distal," which uses 158,803 probes that are >2 kbp 641 from any transcription start site as annotated by GENCODE. ELMER version 2.14 was 642 used with the following parameters: get.diff.meth(sig.diff) $=0$, get.diff.meth(p_value) = 643 0.05, get.diff.meth (minSubgroupFrac) $=0.2$, get.pair $(\mathrm{Pe})=0.1$, get.pair(raw.pvalue) $=$ 644 0.05, get.pair(filter.probes) = FALSE, get.pair(diff.dir)=both, get.pair(permu.size) =100, 645 get.pair(minSubgroupFrac) = 0.4, get.enriched.motif(lower.OR) = 1.1, 646 get.enriched.motif(min.incidence) $=2$. For each comparison, the TF subfamilies were 
647 inferred from the TFs, which had the most significant anticorrelation scores. The 648 candidate MRTFs were next identified within the TF subfamily with FDR $<0.05$ using 649 the classification from TFClass.

\section{Supervised analysis associated with treatment}

651 Only patients with known TMZ and RT treatment status after initial surgery were 652 included in the supervised analysis. In total, we identified 6 patients who received both $653 \mathrm{TMZ}$ and RT, 12 patients who received only $\mathrm{TMZ}, 18$ patients who received RT only and 65433 patients who did not receive additional treatment besides surgery. We used the 655 Kruskal-Wallis test by ranks followed by multiple testing using the Benjamini \& 656 Hochberg $(\mathrm{BH})$ method for false discovery rate (FDR) estimation (Benjamini et al., 657 2001) to identify differentially methylated sites between these four groups at first 658 recurrence. To define significant $\mathrm{CpG}$ probes, we selected probes with FDR $<0.01$ and 659 mean DNA methylation difference $>20 \%$.

660 We investigated the association of differentially methylated CpG probes (FDR $<0.01 ; \mathrm{N}$ $661=5,218 \mathrm{CpG}$ probes) with gene expression from 6 untreated and 14 treated (TMZ, RT 662 and $\mathrm{TMZ}+\mathrm{RT}$ ) first recurrent samples with available DNA methylation and RNA663 sequencing data using ELMER and filtering by distal probes (Silva et al., 2019). Finally, 664 we applied the function "get.enriched.motif" with only "A" motif quality score (highest 665 confidence) to identify CpG-gene pairs enriched for the same DNA motif signature. The 666 CpG probes and genes of interest identified by these analyses were then sent to our 667 collaborators who are part of the GLASS-NL consortium for validation of our results.

669 The GLASS-NL consortium has collected material from $100 \mathrm{IDH}$-mutant astrocytoma 670 (1p19q non-codeleted) patients who underwent at least two surgical resections. Material 671 for analysis had to be available for both resections, and the surgical interval between 672 resections was $>6$ months. Detailed clinical data, imaging, and treatment data of 673 patients was collected within the consortium. All institutions obtained ethics approval 674 from their institutional review boards or ethics review committees before initiation of the 
675 project. All patients provided written informed consent according to local and national 676 guidelines.

677 DNA and RNA were isolated from formalin-fixed paraffin-embedded (FFPE) tumour 678 samples as previously described (Draaisma et al., 2020). Evaluation of the area with 679 highest tumor content was done by the pathologist (PW) on a hematoxylin and eosin 680 stained section. Macrodissection of the marked area was then done on 10-20 10 681 consecutive slides. DNA and RNA extraction was performed using the QIAamp DNA 682 FFPE and RNeasy FFPE kit respectively (both Qiagen, Venlo, The Netherlands). DNA 683 methylation profiling was performed with the Infinium MethylationEPIC BeadChip 684 according to the manufacturer's instructions making use of the Infinium FFPE DNA 685 Restoration Kit. RNA-sequencing was done by Genomescan (Leiden, the Netherlands) 686 and data processing, alignment and further analysis of read counts was done as 687 described (Hoogstrate et al., 2020).

Deconvolution analysis

689 We first constructed a signature matrix from reference DNA methylation profiles of pure 690 flow-sorted populations of cells from the literature. This signature matrix represents a 691 set of differentially methylated CpGs selected and weighted to reflect specificity for a 692 given cell type and is used as the basis of cell deconvolution by methylCIBERSORT. 693 Our final signature matrix consisted of 10 cell types: CD19+ cells (B cells) ( $n=6)$, CD8+ 694 T cells $(n=6)$, CD56+ (natural killer cells) $(n=6)$, and neutrophils $(n=12)$ were from the 695 FlowSorted.Blood.450k Bioconductor package version 1.30.0 (Reinius et al., 2012). 696 CD4+ effector T cells $(n=6)$ and T regs $(n=4)$ were from (Zhang et al., 2013), accessed 697 through the MethyICIBERSORT R package (Chakravarthy et al., 2018). Vascular 698 endothelial cells $(n=2)$ data was from (Moss et al., 2018). Monocyte-derived 699 macrophage $(n=4)$ data was from (Dekkers et al., 2019). Neuron $(n=31)$ and glia cells $700(\mathrm{n}=31)$ were from (Gasparoni et al., 2018). The MethylCIBERSORT R package was 701 used to derive the DNA methylation signature for the deconvolution and the signature 702 matrix was exported and uploaded to the CIBERSORTx portal to be deconvoluted using 7031000 permutations without quantile normalization. 


\section{Validation of tumor cell composition}

Sections of formalin-fixed, paraffin-embedded human glioma surgical samples

were deparaffinized with xylene and rehydrated through graded alcohol into deionized

$708 \mathrm{H}_{2} \mathrm{O}$. Antigens were unmasked by incubation for 45 mins at $95^{\circ} \mathrm{C}$ in Diva Decloaker

709 (Biocare, DV2004) using Biocare's Decloaking chamber, and sections were stained with

710 the antibodies listed on the table, visualized with intelliPATH FLX ${ }^{\text {TM }}$ DAB Chromogen Kit

711 (Biocare, IPK5010) and counterstained with intelliPATH ${ }^{\mathrm{TM}}$ Hematoxylin (Biocare,

712 IPCS5006L). The antibodies are listed in the Key Resource Table.

713 Images of the tissue sections stained by immunohistochemistry using CD163,

714 CD31, and CD8 antibodies were captured by an Olympus IX70 microscope and a digital

715 camera. For quantitative analysis, we selected eight representative areas in each

716 section. Images of the representative areas were captured at a 10X magnification. For

717 CD163 and CD31 stainings, individual cells per area were identified by strong brown

718 stain and counted by using ImageJ ( $\mathrm{NIH}$, Bethesda USA) by an algorithm to evaluate

719 staining using hematoxylin and DAB staining specific built-in color deconvolution plug-

720 in. For CD8 immunostaining, positive cells identified in each area by strong brown stain

721 were manually counted. The cell counting was repeated three times. All images were

722 analyzed in a blinded fashion. Values are presented as mean \pm standard deviation.

723 Groups were compared and potential differences were identified using the non-

724 parametric t test.

725

726 Statistical analysis

727 Data visualization and statistical analysis were performed using $R$ version 4.1.0

728 software packages (www.r-project.org) and Bioconductor (Gentleman et al., 2004).

729 Unless specified, significance was access with an FDR adjustment of less than $5 \%$. 
Code and data availability

732 Processed data for the GLASS consortium is available on Synapse 733 (https://www.synapse.org/\#!Synapse:syn21589818) and will be publicly available on

734 November 9, 2021.

\section{Acknowledgements}

736 This work is supported by the National Institutes of Health under grant numbers 737 R01CA222146 (H.N.), R01CA222146 (I.D., L.M.P.), R01NS096236 (E.G.V.M.), 738 R01NS117666 (E.G.V.M.), R01NS042645 (S.B.), P50CA190991-07 (M.K.); the 739 Department of Defense grant number CA170278 (T.S.S., L.M.P., H.N.); São Paulo 740 Research Foundation (FAPESP) grant numbers 2018/00583-0 and 2019/14928-1

741 (T.M.M.); Leeds Cares grant [9R11/14-11] and the Sidney Driscol Neuroscience 742 Foundation Contribution to Brain Tumour Northwest (L.F.S.); University of Colorado 743 Department of Neurosurgery Nervous System Biorepository (D.R.O.); FNR CORE 744 C20/BM/14646004 (GLASS-LUX) and FNRS-Televie TETHER (S.P.N.); FNRS-Televie 745 TETHER (A.C.H.); F.S.V. is supported by the JAX Scholar Program and a postdoctoral 746 fellowship from The Jane Coffin Childs Memorial Fund for Medical Research; GLASS-

747 NL was supported by the Dutch Cancer Society KWF grant number 11026 (B.Y., 748 B.A.W., P.W., M.C.M.K., W.V., P.J.F., M.J.V.D.B., J.M.N., M.S.). 


\begin{tabular}{|c|c|c|}
\hline REAGENT or RESOURCE & SOURCE & IDENTIFIER \\
\hline \multicolumn{3}{|l|}{ Software and Algorithms } \\
\hline MethylCIBERSORT & (Chakravarthy et al., 2018) & $\begin{array}{l}\text { https://doi.org/10.5281/zen } \\
\text { odo.1298968 }\end{array}$ \\
\hline CIBERSORTX & (Newman et al., 2019) & $\begin{array}{l}\text { https://cibersortx.stanford.e } \\
\text { du/ }\end{array}$ \\
\hline$R$ version 4.0 .5 & (R Core Team, 2017) & https://www.R-project.org \\
\hline $\operatorname{minfi}(\mathrm{v} 1.36 .0)$ & (Aryee et al., 2014) & $\begin{array}{l}\text { https://bioconductor.org/pa } \\
\text { ckages/release/bioc/html/ } \\
\text { minfi.html }\end{array}$ \\
\hline TCGAbiolinks (v2.18.0) & (Colaprico et al., 2016) & $\begin{array}{l}\text { http://bioconductor.org/pac } \\
\text { kages/TCGAbiolinks/ }\end{array}$ \\
\hline ELMER (v2.14.0) & (Silva et al., 2019) & $\begin{array}{l}\text { http://bioconductor.org/pac } \\
\text { kages/ELMER/ }\end{array}$ \\
\hline $\begin{array}{l}\text { DNA methylation-based } \\
\text { classification of central } \\
\text { nervous system tumors }\end{array}$ & (Capper et al., 2018) & $\begin{array}{l}\text { https://www.molecularneur } \\
\text { opathology.org/mnp }\end{array}$ \\
\hline CD8 & $\begin{array}{l}\text { Rabbit Monoclonal } \\
\text { (C8/1779R) }\end{array}$ & Biocare, API3219AA \\
\hline CD31 & Mouse Monoclonal (BC2) & Biocare, PM347 \\
\hline CD163 & Mouse Monoclonal (10D6) & Biocare, PM353AA \\
\hline \multicolumn{3}{|l|}{ Deposited Data } \\
\hline Raw DNA methylation data & This paper & \\
\hline TCGA data & $\begin{array}{l}\text { NIH Genomic Data } \\
\text { Commons (GDC) }\end{array}$ & $\begin{array}{l}\text { https://gdc.cancer.gov/abo } \\
\text { ut- } \\
\text { data/publications/PanCan } \\
\text { Stemness-2018 }\end{array}$ \\
\hline $\begin{array}{l}\text { Published longitudinal } \\
\text { glioma cohort }\end{array}$ & (Mazor et al., 2015) & EGAS00001001255 \\
\hline
\end{tabular}




\begin{tabular}{|l|l|l|}
\hline $\begin{array}{l}\text { Published longitudinal } \\
\text { glioma cohort }\end{array}$ & (Mazor et al., 2017) & EGAS00001001854 \\
\hline $\begin{array}{l}\text { Published longitudinal } \\
\text { glioma cohort }\end{array}$ & (Bai et al., 2016) & EGAS00001001588 \\
\hline Neuron and glia cells data & (Gasparoni et al., 2018) & GSE66351 \\
\hline $\begin{array}{l}\text { Blood cells (CD19+, CD8+ } \\
\text { T, CD56+, neutrophils }\end{array}$ & (Reinius et al., 2012) & GSE35069 \\
\hline CD4+ effector T and T regs & (Zhang et al., 2013) & GSE49667 \\
\hline Vascular endothelial cells & (Moss et al., 2018) & GSE122126 \\
\hline $\begin{array}{l}\text { Monocyte-derived } \\
\text { macrophage }\end{array}$ & (Dekkers et al., 2019) & GSE118696 \\
\hline
\end{tabular}




\section{Author Contributions:}

Conceptualization, T.M.M., T.S.S., R.G.W.V., A.I., H.N.; Investigation, T.M.M., T.S.S.;

762 Resources, T.M.M., T.S.S., F.S.V.;

763 Data curation, T.M.M, T.S.S, I.D., F.S.V., L.G., W.V., K.A., L.D., S.B., J.S.B., H.K.G., M.H., A.C.H., K.C.J, M.K., E.K., M.C.M.K., S.M., S.P.N., J.M.N., D.R.O., S.H.P., G.R., P.A.S.S., M.S., L.F.S., M.J.V.D.B., E.G.V.M., A.W., T.W., M.W., B.A.W., B.Y., P.W., A.L., P.J.F., L.M.P., R.G.W.V., A.I., H.N.; Writing- Original draft, T.M.M., T.S.S., H.N., A.I.; Writing-Review \& Editing, T.M.M, T.S.S, I.D., F.S.V., L.G., W.V., K.A., L.D., S.B., J.S.B., H.K.G., M.H., A.C.H., K.C.J, M.K., E.K., M.C.M.K., S.M., S.P.N., J.M.N., D.R.O., S.H.P., G.R., P.A.S.S., M.S., L.F.S., M.J.V.D.B., E.G.V.M., A.W., T.W., M.W., B.A.W., B.Y., P.W., A.L., P.J.F., L.M.P., R.G.W.V., A.I., H.N.;

772 Funding acquisition, H.N., L.M.P., R.G.W.V.;

773 Supervision, H.N., A.I., R.G.W.V.

All co-authors and contributors discussed the results and commented on the manuscript.

776 


\section{Bibliography}

Aryee, M.J., Jaffe, A.E., Corrada-Bravo, H., Ladd-Acosta, C., Feinberg, A.P., Hansen, K.D., and Irizarry, R.A. (2014). Minfi: a flexible and comprehensive Bioconductor package for the analysis of Infinium DNA methylation microarrays. Bioinformatics 30, 1363-1369.

Bai, H., Harmancı, A.S., Erson-Omay, E.Z., Li, J., Coşkun, S., Simon, M., Krischek, B., IDH1-mutant glioma malignant progression. Nat. Genet. 48, 59-66.

Barthel, F.P., Johnson, K.C., Varn, F.S., Moskalik, A.D., Tanner, G., Kocakavuk, E., Anderson, K.J., Abiola, O., Aldape, K., Alfaro, K.D., et al. (2019). Longitudinal molecular trajectories of diffuse glioma in adults. Nature 576, 112-120.

805 Baumert, B.G., Hegi, M.E., van den Bent, M.J., von Deimling, A., Gorlia, T., Hoang-Xuan, K., Brandes, A.A., Kantor, G., Taphoorn, M.J.B., Hassel, M.B., et al. (2016). Temozolomide chemotherapy versus radiotherapy in high-risk low-grade glioma (EORTC 22033-26033): a randomised, open-label, phase 3 intergroup study. Lancet Oncol. 17, 1521-1532.

Benjamini, Y., Drai, D., Elmer, G., Kafkafi, N., and Golani, I. (2001). Controlling the false discovery rate in behavior genetics research. Behav. Brain Res. 125, 279-284. K., and Vardimon, L. (2012). Aberrant expression of C-Jun in glioblastoma by internal ribosome entry site (IRES)-mediated translational activation. Proc Natl Acad Sci USA 109, E2875-84. Brat, D.J., Aldape, K., Colman, H., Figrarella-Branger, D., Fuller, G.N., Giannini, C., Holland, E.C., Jenkins, R.B., Kleinschmidt-DeMasters, B., Komori, T., et al. (2020). cIMPACT-NOW update 5: recommended grading criteria and terminologies for IDH-mutant astrocytomas. Acta Neuropathol. 139, 603-608. Ricci, P., Bullard, D., Brown, P.D., et al. (2016). Radiation plus Procarbazine, CCNU, and Vincristine in Low-Grade Glioma. N. Engl. J. Med. 374, 1344-1355.

Capper, D., Jones, D.T.W., Sill, M., Hovestadt, V., Schrimpf, D., Sturm, D., Koelsche, C., Sahm, F., Chavez, L., Reuss, D.E., et al. (2018). DNA methylation-based classification of central nervous system tumours. Nature 555, 469-474.

Ceccarelli, M., Barthel, F.P., Malta, T.M., Sabedot, T.S., Salama, S.R., Murray, B.A., Morozova, O., Newton, Y., Radenbaugh, A., Pagnotta, S.M., et al. (2016). Molecular profiling reveals biologically discrete subsets and pathways of progression in diffuse glioma. Cell 164, 550-563. using DNA methylation. Nat. Commun. 9, 3220. 
830 Colaprico, A., Silva, T.C., Olsen, C., Garofano, L., Cava, C., Garolini, D., Sabedot, T.S., Malta, T.M., Pagnotta, S.M., Castiglioni, I., et al. (2016). TCGAbiolinks: an R/Bioconductor package for integrative analysis of TCGA data. Nucleic Acids Res. 44, e71.

833 Dai, J., Punchihewa, C., Mistry, P., Ooi, A.T., and Yang, D. (2004). Novel DNA bis-intercalation by MLN944, a potent clinical bisphenazine anticancer drug. J. Biol. Chem. 279, 46096-46103.

Dekkers, K.F., Neele, A.E., Jukema, J.W., Heijmans, B.T., and de Winther, M.P.J. (2019). Human monocyte-to-macrophage differentiation involves highly localized gain and loss of DNA methylation at transcription factor binding sites. Epigenetics Chromatin 12, 34.

838 Draaisma, K., Chatzipli, A., Taphoorn, M., Kerkhof, M., Weyerbrock, A., Sanson, M., Hoeben, A., Lukacova, S., Lombardi, G., Leenstra, S., et al. (2020). Molecular Evolution of IDH WildType Glioblastomas Treated With Standard of Care Affects Survival and Design of Precision Medicine Trials: A Report From the EORTC 1542 Study. J. Clin. Oncol. 38, 81-99.

842 Frattini, V., Pagnotta, S.M., Tala, Fan, J.J., Russo, M.V., Lee, S.B., Garofano, L., Zhang, J., Shi, P., Lewis, G., et al. (2018). A metabolic function of FGFR3-TACC3 gene fusions in cancer. Nature 553, 222-227.

845 Fortin, J.-P., Triche, T.J., and Hansen, K.D. (2017). Preprocessing, normalization and 846 integration of the Illumina HumanMethylationEPIC array with minfi. Bioinformatics 33, 558-560.

847 Garofano, L., Migliozzi, S., Oh, Y.T., D’Angelo, F., Najac, R.D., Ko, A., Frangaj, B., Caruso, 848 F.P., Yu, K., Yuan, J., et al. (2021). Pathway-based classification of glioblastoma uncovers a 849 mitochondrial subtype with therapeutic vulnerabilities. Nat. Cancer 2, 141-156.

850 Gasparoni, G., Bultmann, S., Lutsik, P., Kraus, T.F.J., Sordon, S., Vlcek, J., Dietinger, V., 851 Steinmaurer, M., Haider, M., Mulholland, C.B., et al. (2018). DNA methylation analysis on 852 purified neurons and glia dissects age and Alzheimer's disease-specific changes in the human 853 cortex. Epigenetics Chromatin 11, 41.

854 Gentleman, R.C., Carey, V.J., Bates, D.M., Bolstad, B., Dettling, M., Dudoit, S., Ellis, B., 855 Gautier, L., Ge, Y., Gentry, J., et al. (2004). Bioconductor: open software development for 856 computational biology and bioinformatics. Genome Biol. 5, R80.

857 GLASS Consortium (2018). Glioma through the looking GLASS: molecular evolution of diffuse 858 gliomas and the Glioma Longitudinal Analysis Consortium. Neuro Oncol. 20, 873-884.

859 Heiss, J.A., and Just, A.C. (2018). Identifying mislabeled and contaminated DNA methylation microarray data: an extended quality control toolset with examples from GEO. Clin. Epigenetics 10, 73.

Hoogstrate, Y., Vallentgoed, W., Kros, J.M., de Heer, I., de Wit, M., Eoli, M., Sepulveda, J.M., Walenkamp, A.M.E., Frenel, J.-S., Franceschi, E., et al. (2020). EGFR mutations are associated with response to depatux-m in combination with temozolomide and result in a receptor that is hypersensitive to ligand. Neurooncol Adv 2, vdz051. Hunter, C., Smith, R., Cahill, D.P., Stephens, P., Stevens, C., Teague, J., Greenman, C., 
Johnson, B.E., Mazor, T., Hong, C., Barnes, M., Aihara, K., McLean, C.Y., Fouse, S.D., Yamamoto, S., Ueda, H., Tatsuno, K., et al. (2014). Mutational analysis reveals the origin and therapy-driven evolution of recurrent glioma. Science 343, 189-193.

873 Kocakavuk, E., Anderson, K.J., Varn, F.S., Johnson, K.C., Amin, S.B., Sulman, E.P., Lolkema, M.P., Barthel, F.P., and Verhaak, R.G.W. (2021). Radiotherapy is associated with a deletion signature that contributes to poor outcomes in patients with cancer. Nat. Genet. 53, 1088-1096. Leek, J.T., Johnson, W.E., Parker, H.S., Jaffe, A.E., and Storey, J.D. (2012). The sva package for removing batch effects and other unwanted variation in high-throughput experiments. Bioinformatics 28, 882-883. J., et al. (2016). c-Fos over-expression promotes radioresistance and predicts poor prognosis in malignant glioma. Oncotarget 7, 65946-65956.

Louis, D.N., Perry, A., Reifenberger, G., von Deimling, A., Figarella-Branger, D., Cavenee, W.K., Ohgaki, H., Wiestler, O.D., Kleihues, P., and Ellison, D.W. (2016). The 2016 World Health Organization Classification of Tumors of the Central Nervous System: a summary. Acta Neuropathol. 131, 803-820. Louis, D.N., Perry, A., Wesseling, P., Brat, D.J., Cree, I.A., Figarella-Branger, D., Hawkins, C., Ng, H.K., Pfister, S.M., Reifenberger, G., et al. (2021). The 2021 WHO Classification of Tumors of the Central Nervous System: a summary. Neuro Oncol. Malta, T.M., de Souza, C.F., Sabedot, T.S., Silva, T.C., Mosella, M.S., Kalkanis, S.N., Snyder, J., Castro, A.V.B., and Noushmehr, H. (2018a). Glioma CpG island methylator phenotype (GCIMP): biological and clinical implications. Neuro Oncol. 20, 608-620.

Malta, T.M., Sokolov, A., Gentles, A.J., Burzykowski, T., Poisson, L., Weinstein, J.N., Kamińska, B., Huelsken, J., Omberg, L., Gevaert, O., et al. (2018b). Machine Learning Identifies Stemness Features Associated with Oncogenic Dedifferentiation. Cell 173, 338-354.e15.

Mathas, S., Janz, M., Hummel, F., Hummel, M., Wollert-Wulf, B., Lusatis, S., Anagnostopoulos, I., Lietz, A., Sigvardsson, M., Jundt, F., et al. (2006). Intrinsic inhibition of transcription factor E2A by HLH proteins ABF-1 and Id2 mediates reprogramming of neoplastic $B$ cells in Hodgkin lymphoma. Nat. Immunol. 7, 207-215.

Mathur, R., Zhang, Y., Grimmer, M.R., Hong, C., Zhang, M., Bollam, S., Petrecca, K., Clarke, J., Berger, M.S., Phillips, J.J., et al. (2020). MGMT promoter methylation level in newly diagnosed low-grade glioma is a predictor of hypermutation at recurrence. Neuro Oncol. 22, 1580-1590.

908 Mazor, T., Pankov, A., Johnson, B.E., Hong, C., Hamilton, E.G., Bell, R.J.A., Smirnov, I.V., Reis, G.F., Phillips, J.J., Barnes, M.J., et al. (2015). DNA methylation and somatic mutations converge on the cell cycle and define similar evolutionary histories in brain tumors. Cancer Cell 28, 307-317.

909

Mazor, T., Chesnelong, C., Pankov, A., Jalbert, L.E., Hong, C., Hayes, J., Smirnov, I.V., Marshall, R., Souza, C.F., Shen, Y., et al. (2017). Clonal expansion and epigenetic reprogramming following deletion or amplification of mutant IDH1. Proc Natl Acad Sci USA 114, 10743-10748. 
Moss, J., Magenheim, J., Neiman, D., Zemmour, H., Loyfer, N., Korach, A., Samet, Y., Maoz, M., Druid, H., Arner, P., et al. (2018). Comprehensive human cell-type methylation atlas reveals origins of circulating cell-free DNA in health and disease. Nat. Commun. 9, 5068. Newman, A.M., Steen, C.B., Liu, C.L., Gentles, A.J., Chaudhuri, A.A., Scherer, F., Khodadoust, M.S., Esfahani, M.S., Luca, B.A., Steiner, D., et al. (2019). Determining cell type abundance and expression from bulk tissues with digital cytometry. Nat. Biotechnol. 37, 773-782. Ng, D., Pitcher, G.M., Szilard, R.K., Sertié, A., Kanisek, M., Clapcote, S.J., Lipina, T., Kalia, L.V., Joo, D., McKerlie, C., et al. (2009). Neto1 is a novel CUB-domain NMDA receptorinteracting protein required for synaptic plasticity and learning. PLoS Biol. 7, e41. Nomura, M., Saito, K., Aihara, K., Nagae, G., Yamamoto, S., Tatsuno, K., Ueda, H., Fukuda, S., Umeda, T., Tanaka, S., et al. (2019). DNA demethylation is associated with malignant progression of lower-grade gliomas. Sci. Rep. 9, 1903.

Noushmehr, H., Weisenberger, D.J., Diefes, K., Phillips, H.S., Pujara, K., Berman, B.P., Pan, F., Pelloski, C.E., Sulman, E.P., Bhat, K.P., et al. (2010). Identification of a CpG island methylator phenotype that defines a distinct subgroup of glioma. Cancer Cell 17, 510-522.

Reinius, L.E., Acevedo, N., Joerink, M., Pershagen, G., Dahlén, S.-E., Greco, D., Söderhäll, C., Scheynius, A., and Kere, J. (2012). Differential DNA methylation in purified human blood cells: implications for cell lineage and studies on disease susceptibility. PLoS ONE 7, e41361.

R Core Team, R.C.T. (2017). R: A language and environment for statistical computing (Vienna, Austria: R Foundation for Statistical Computing). A., Reinhardt, A., Huang, K., et al. (2018). Novel, improved grading system(s) for IDH-mutant astrocytic gliomas. Acta Neuropathol. 136, 153-166.

Silva, T.C., Coetzee, S.G., Gull, N., Yao, L., Hazelett, D.J., Noushmehr, H., Lin, D.-C., and Berman, B.P. (2019). ELMER v.2: an R/Bioconductor package to reconstruct gene regulatory networks from DNA methylation and transcriptome profiles. Bioinformatics 35, 1974-1977. de Souza, C.F., Sabedot, T.S., Malta, T.M., Stetson, L., Morozova, O., Sokolov, A., Laird, P.W., Wiznerowicz, M., lavarone, A., Snyder, J., et al. (2018). A Distinct DNA Methylation Shift in a Subset of Glioma CpG Island Methylator Phenotypes during Tumor Recurrence. Cell Rep. 23, 637-651.

Tesileanu, C.M.S., van den Bent, M.J., Sanson, M., Wick, W., Brandes, A.A., Clement, P.M., Erridge, S.C., Vogelbaum, M.A., Nowak, A.K., Baurain, J.F., et al. (2021). Prognostic significance of genome-wide DNA methylation profiles within the randomised, phase 3, EORTC CATNON trial on non-1p/19q deleted anaplastic glioma. Neuro Oncol.

Touat, M., Li, Y.Y., Boynton, A.N., Spurr, L.F., Iorgulescu, J.B., Bohrson, C.L., Cortes-Ciriano, I., Birzu, C., Geduldig, J.E., Pelton, K., et al. (2020). Mechanisms and therapeutic implications of hypermutation in gliomas. Nature 580, 517-523.

Triche, T.J., Weisenberger, D.J., Van Den Berg, D., Laird, P.W., and Siegmund, K.D. (2013). Low-level processing of Illumina Infinium DNA Methylation BeadArrays. Nucleic Acids Res. 41, e90. 
950 Turcan, S., Rohle, D., Goenka, A., Walsh, L.A., Fang, F., Yilmaz, E., Campos, C., Fabius, 951 A.W.M., Lu, C., Ward, P.S., et al. (2012). IDH1 mutation is sufficient to establish the glioma 952 hypermethylator phenotype. Nature 483, 479-483.

953 van den Bent, M.J., Afra, D., de Witte, O., Ben Hassel, M., Schraub, S., Hoang-Xuan, K., 954 Malmström, P.O., Collette, L., Piérart, M., Mirimanoff, R., et al. (2005). Long-term efficacy of 955 early versus delayed radiotherapy for low-grade astrocytoma and oligodendroglioma in adults: 956 the EORTC 22845 randomised trial. Lancet 366, 985-990.

957 Varn, F.S., Johnson, K.C., Wade, T.E., Malta, T.M., Sabedot, T.S., Barthel, F.P., Kim, H., 958 Ahmed, N., Datta, I., Barnholtz-Sloan, J.S., et al. (2021). Longitudinal analysis of diffuse glioma 959 reveals cell state dynamics at recurrence associated with changes in genetics and the 960 microenvironment. BioRxiv.

961 Weller, M., van den Bent, M., Preusser, M., Le Rhun, E., Tonn, J.C., Minniti, G., Bendszus, M., 962 Balana, C., Chinot, O., Dirven, L., et al. (2021). EANO guidelines on the diagnosis and 963 treatment of diffuse gliomas of adulthood. Nat. Rev. Clin. Oncol. 18, 170-186.

964 Wick, W., Hartmann, C., Engel, C., Stoffels, M., Felsberg, J., Stockhammer, F., Sabel, M.C., 965 Koeppen, S., Ketter, R., Meyermann, R., et al. (2009). NOA-04 randomized phase III trial of 966 sequential radiochemotherapy of anaplastic glioma with procarbazine, lomustine, and vincristine 967 or temozolomide. J. Clin. Oncol. 27, 5874-5880.

968 Wu, X., Liu, Y., Chen, X., Sun, Q., Tang, R., Wang, W., Yu, Z., and Xie, M. (2013). Involvement of TREK-1 activity in astrocyte function and neuroprotection under simulated ischemia

971 Yao, L., Shen, H., Laird, P.W., Farnham, P.J., and Berman, B.P. (2015). Inferring regulatory 972 element landscapes and transcription factor networks from cancer methylomes. Genome Biol.

$97316,105$.

974 Yu, L., Mikloucich, J., Sangster, N., Perez, A., and McCormick, P.J. (2003). MyoR is expressed 975 in nonmyogenic cells and can inhibit their differentiation. Exp. Cell Res. 289, 162-173.

976 Zhang, Y., Maksimovic, J., Naselli, G., Qian, J., Chopin, M., Blewitt, M.E., Oshlack, A., and 977 Harrison, L.C. (2013). Genome-wide DNA methylation analysis identifies hypomethylated genes 978 regulated by FOXP3 in human regulatory T cells. Blood 122, 2823-2836. 
Molecular subtype switch vs. Hypermutation
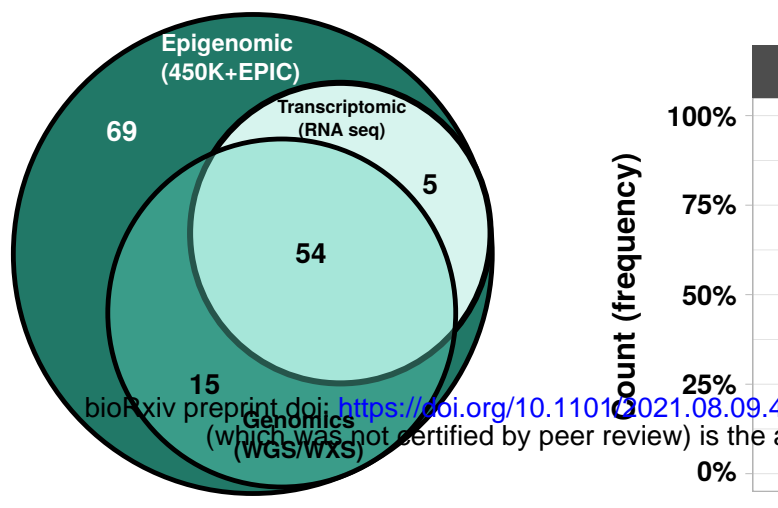

\section{IDH Mut}
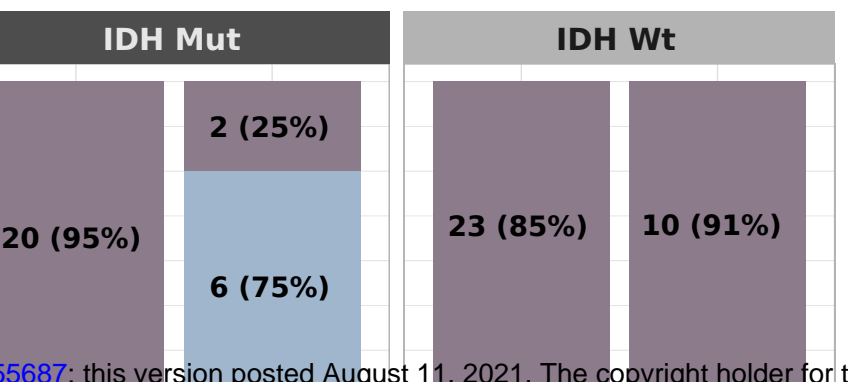

Hypermutator

$23(85 \%)$

$10(91 \%)$

status

B

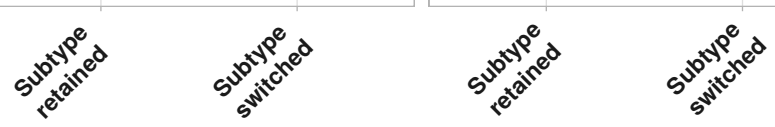

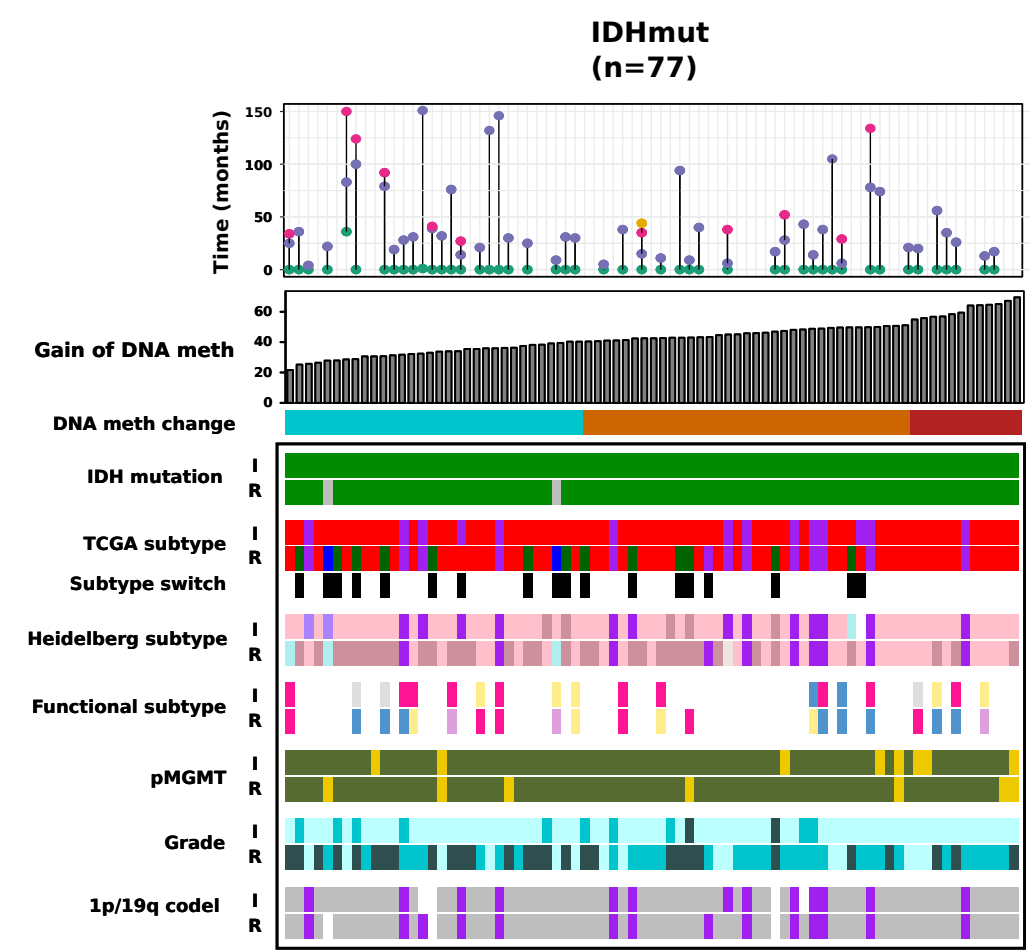

IDHwt

IDHmut

$(n=66)$
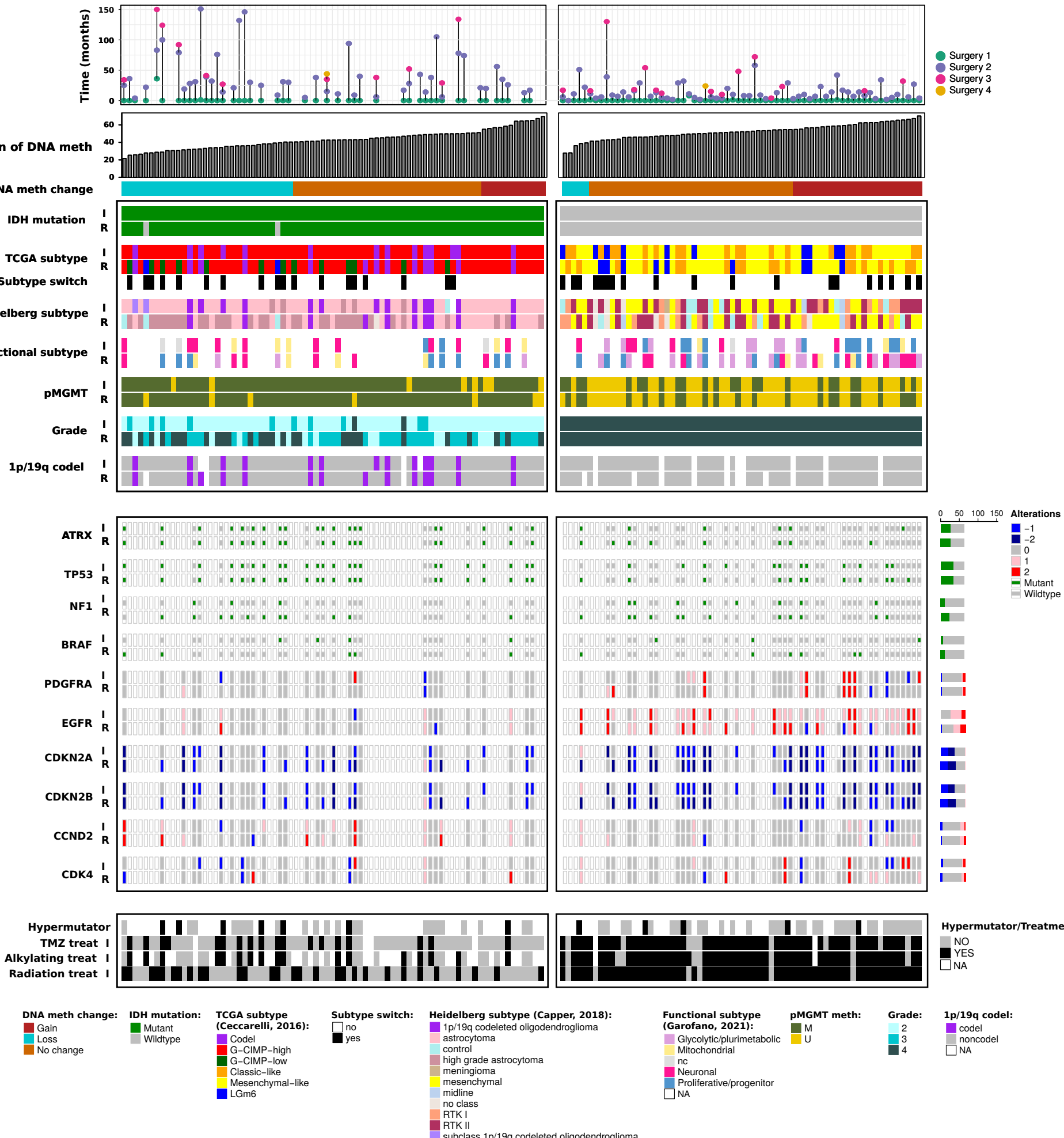

Figure 1. Epigenomic evolution of matched initial and recurrent gliomas. A) Venn diagram of number of patients who had DNA methylation, genomic (WGS/WXS) and/or RNAseq profiling. B) Clinical and molecular overview of matched initial and recurrent DNA methylation cohort. Each column represents a single patient $(n=143)$ at two separate time points grouped by IDH status and ordered by increase of gain of DNA methylation at recurrent tumor from left-to-right. Top plot shows the surgical interval of each patient. C) Frequency of patients with hypermutator tumors that switched or retained molecular subtype at recurrence. Patients are divided by IDH status. 

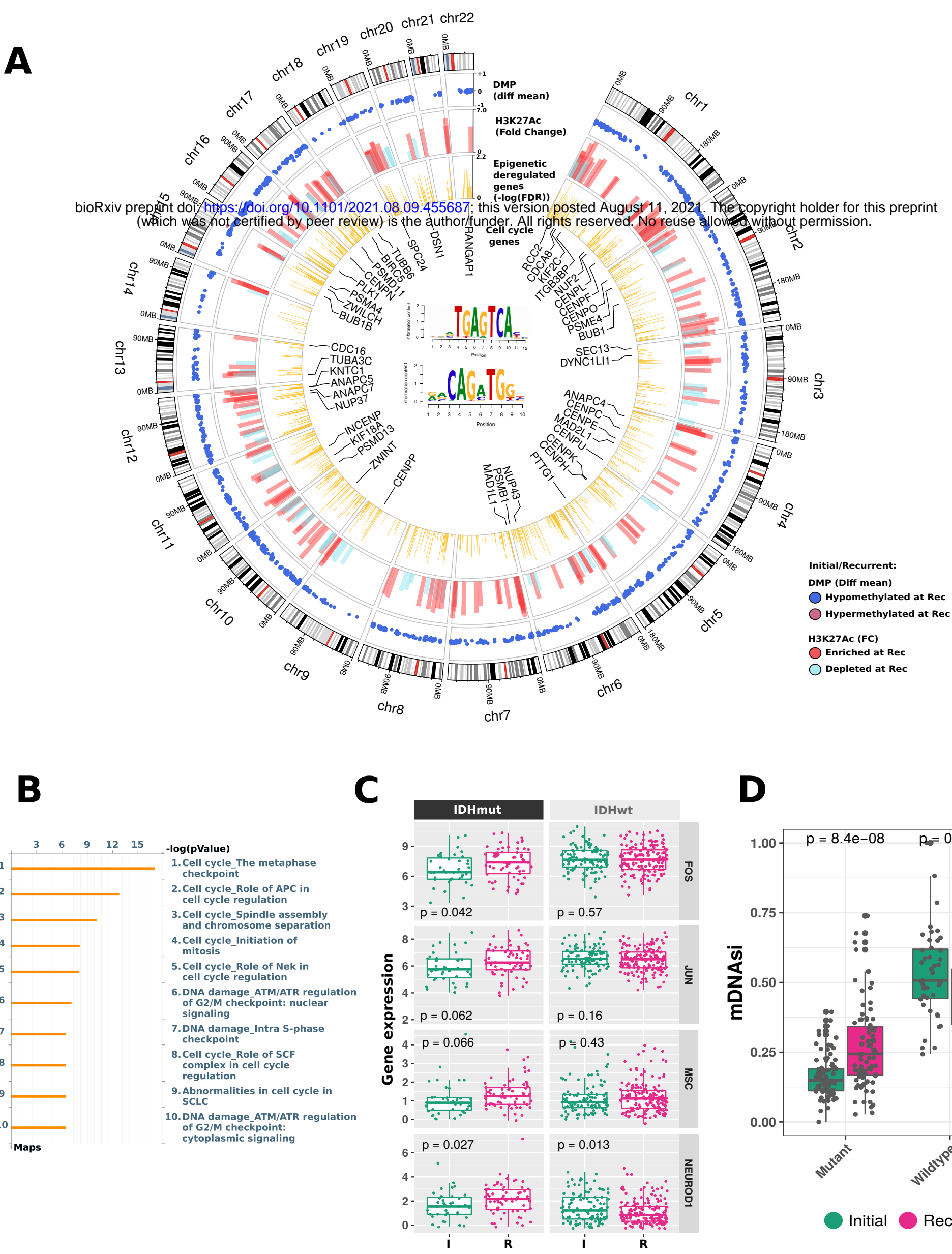

D

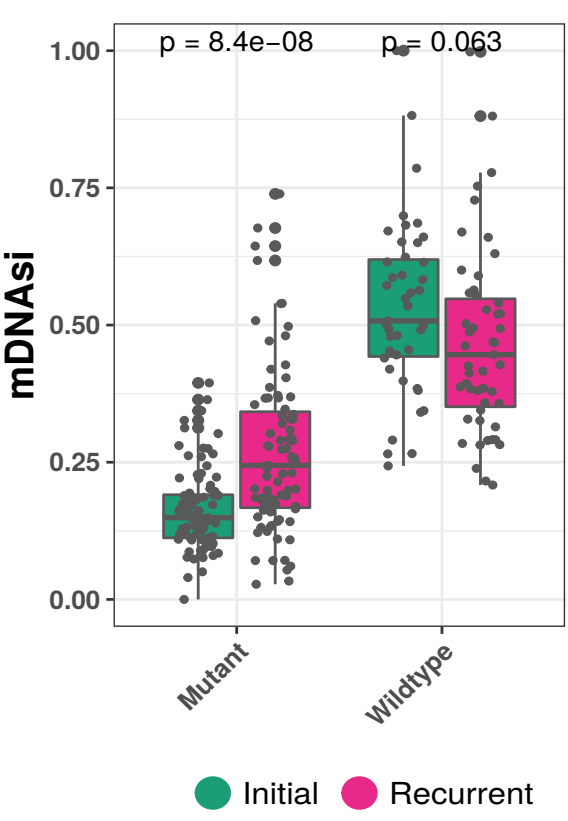

Figure 2. Master regulators associated with IDHmut glioma recurrence/progression. A) Circos plot diagram showing epigenomic changes between initial and recurrent IDHmut tumors. The outer track shows chromosome location. The second track shows genomic location of 1,590 differentially methylated $\mathrm{CpG}$ probes between initial and recurrent IDHmut tumors. Height of dots represents the difference of methylation levels between recurrent and initial. The third track shows the genomic location of $\mathrm{H} 3 \mathrm{H} 27 \mathrm{ac}$ peaks defined by differential binding analysis of IDHmut G-CIMP-high patients that progressed to G-CIMP-low. Height of the peaks represents the fold change of differential binding analysis. The fourth inner track shows the genomic location of the candidate genes epigenetically regulated by the CpG probes (yellow bars). Height of the bars represents the -log of FDR. Genes related to cell cycle are labeled. The top transcription factor (TF) binding motifs enriched among the candidate DMP are FOS/JUN family and NEUROD1, whose DNA signature motifs are represented in the center of the circos plot. B) Pathway analysis of epigenetically activated genes in recurrent IDHmut tumors. C) Gene expression levels of master regulator transcription factors (MRTFs) of IDHmut initial and recurrent tumors. IDHwt tumors are shown as a reference. D) Stemness activity in glioma samples stratified by primary/recurrent and IDH mutation status. 


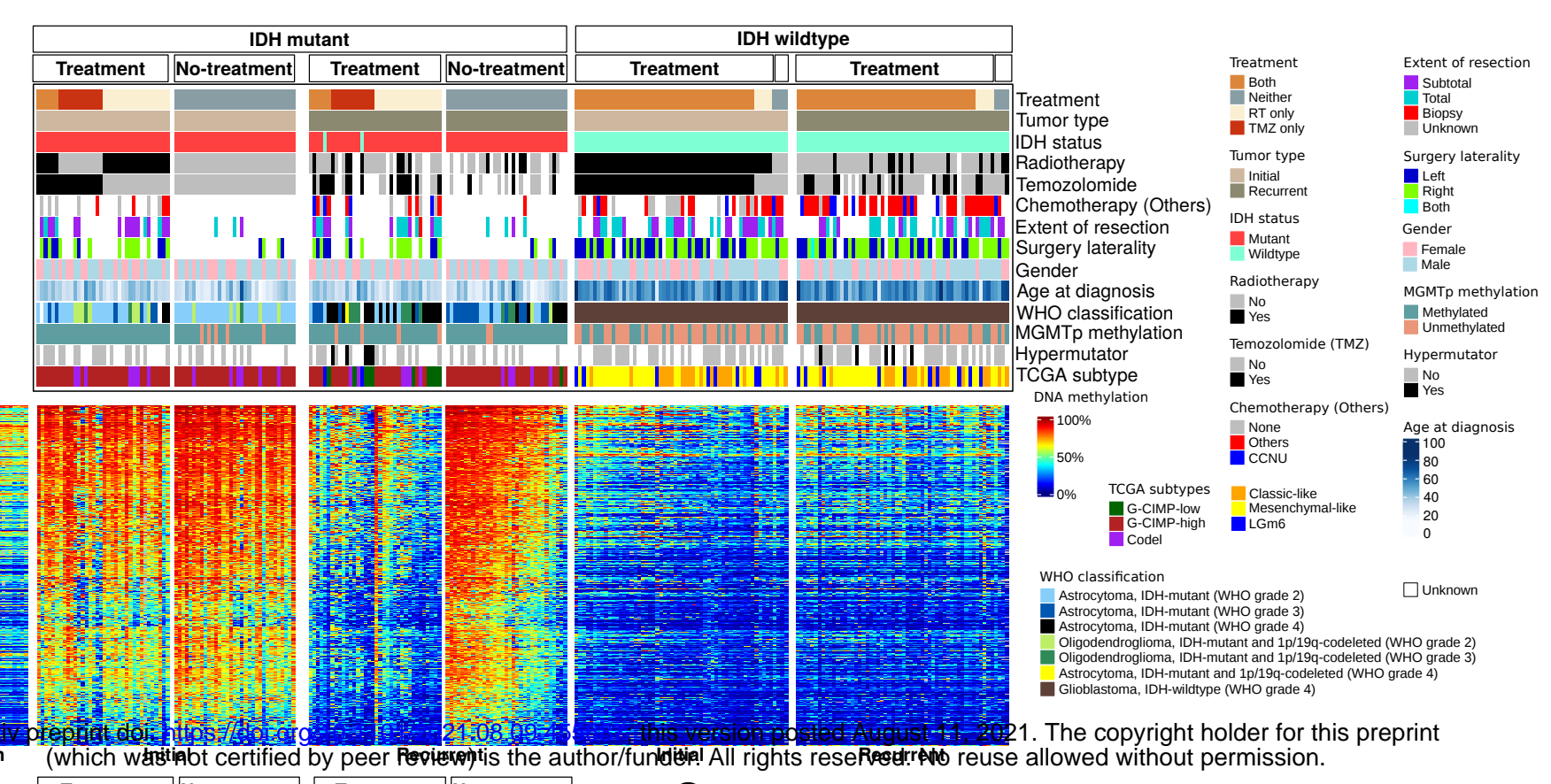

B
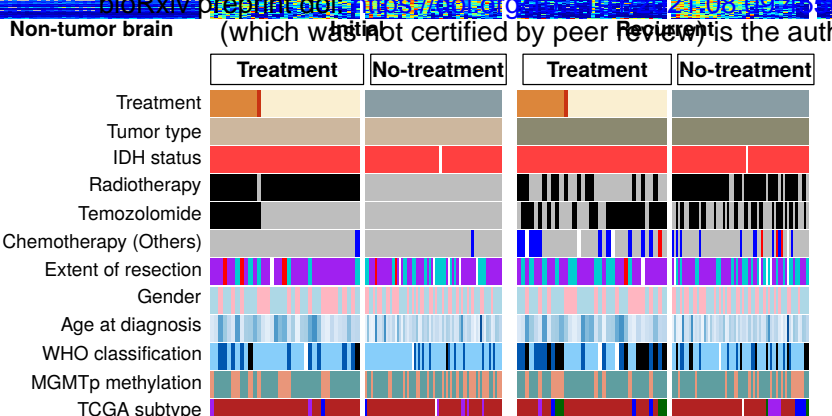

TCGA subtype
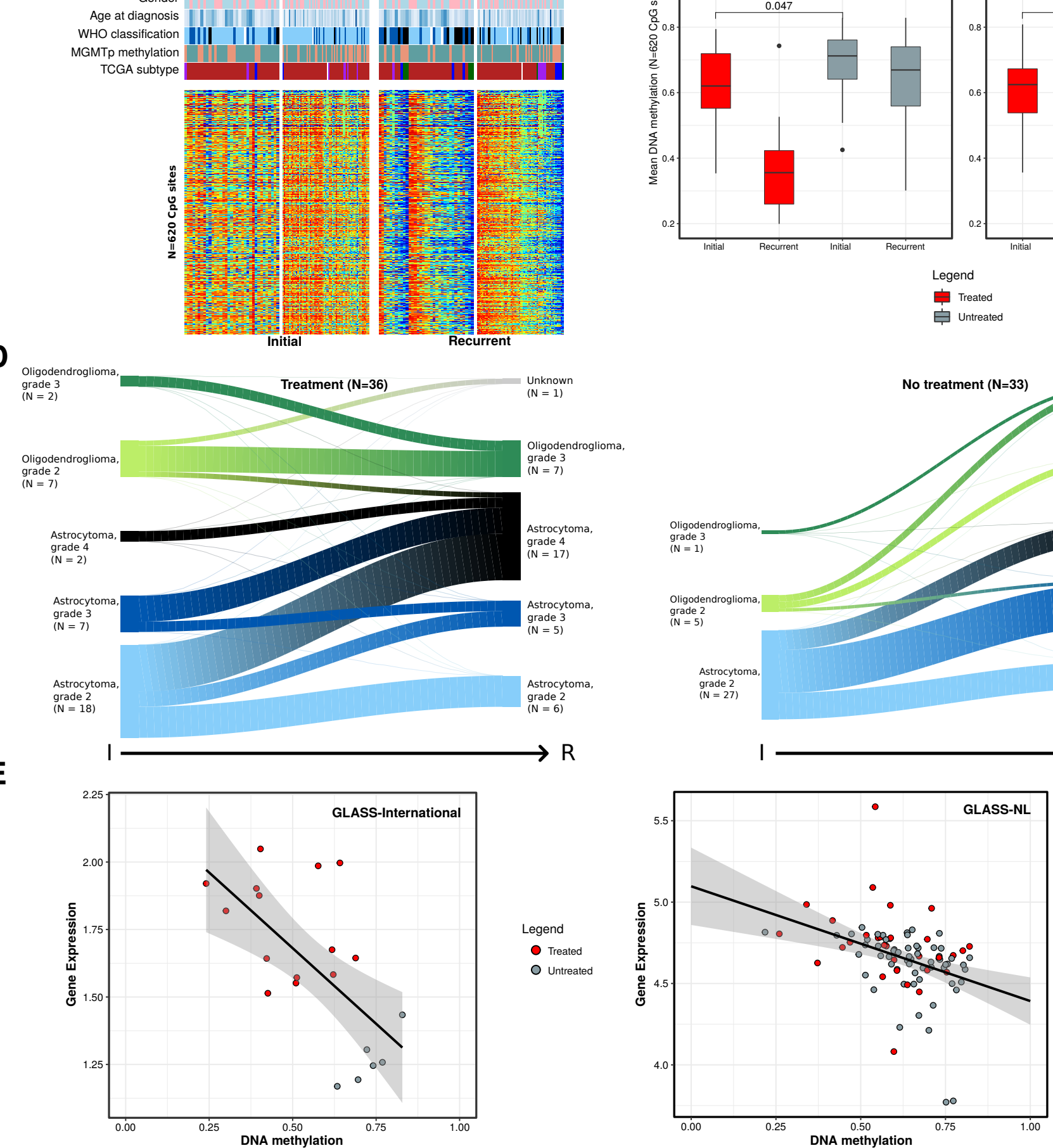

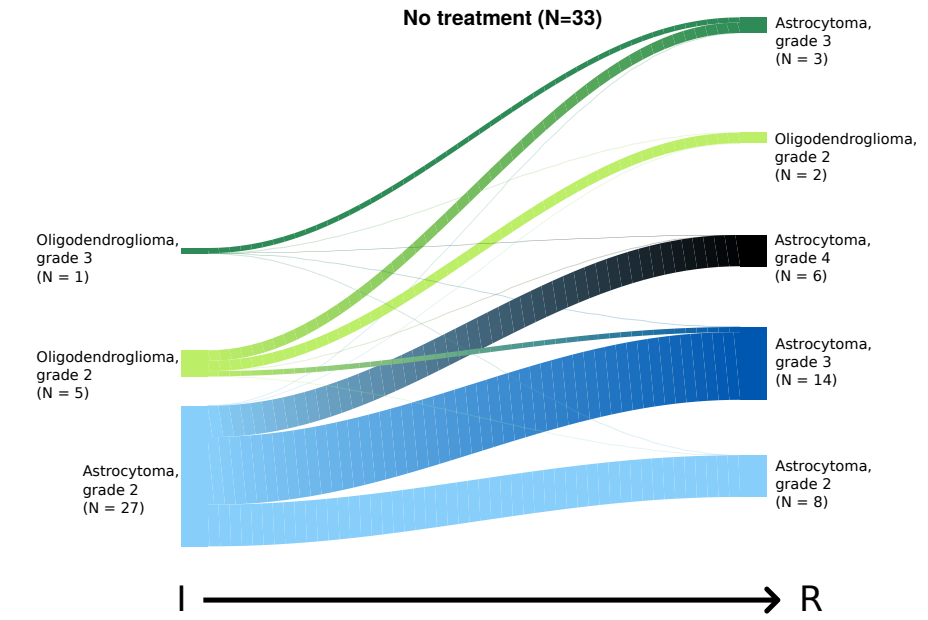

Figure 3. DNA methylation loss associates with malignant transformation of glioma after standard

treatment. A) Heatmap of DNA methylation data. Supervised hierarchical clustering analysis of 620 CpG probes that are associated with different treatment strategies in IDH-mutant paired glioma samples. Columns represent glioma samples, rows represent CpG probes. Samples were stratified and clustered based on IDH mutation status and primary/recurrent status and CpGs were ordered using hierarchical clustering methods. Non-neoplastic brain samples are represented on the left of the heatmap. DNA methylation beta-values range from 0 (low) to 1 (high). Additional tracks are included at the top of the heatmaps to identify each sample membership within separate cluster analysis. B) Heatmap of DNA methylation data in the validation cohort - GLASS-NL, showing the same 620 CpG probes of panel A. C) Boxplot of the average DNA methylation beta-value of the $620 \mathrm{CpG}$ probes from panel A. Samples are stratified by primary/recurrent status and by treated/non-treated status. Left: GLASS-International samples. Right: GLASS-NL samples. D) Evolution of tumor histology (2021 WHO classification) from primary to recurrent samples after treatment compared to non-treated gliomas. E) Scatter plot of mean DNA methylation of CpG probes and mean gene expression of the epigenetically regulated genes after treatment. 


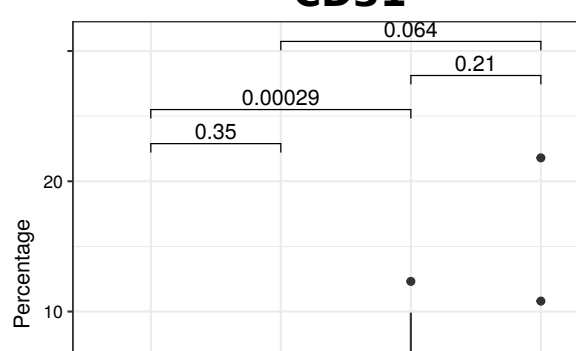

B

bioRxiv preprin? doi: htps:/ddei org/10.1101/2021 08 09.455687 this version posted August 11, 2021. The copyright holder for this preprint (which was not certified by peer review) is the author/funder. All rights reserved. No reyse all

Initial Initial Recurrent Recurrent

\section{CD8}

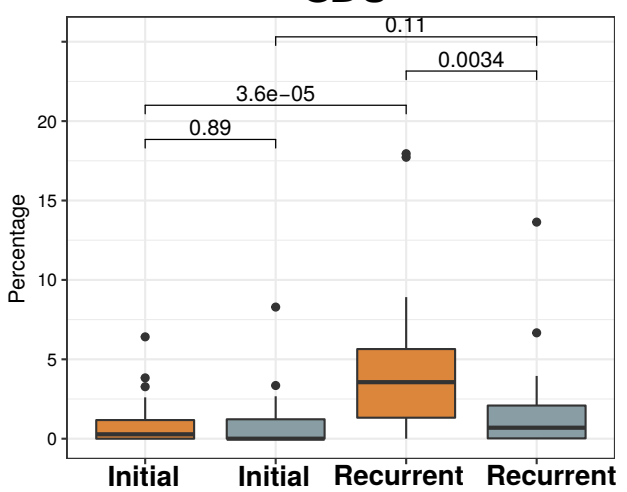

Legend:

追 Treated

Untreated

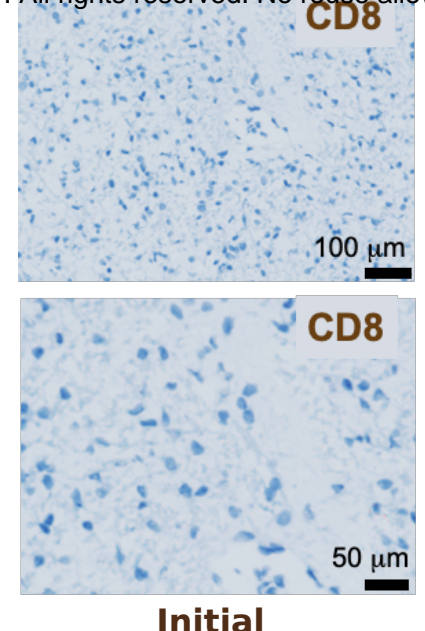

Initial

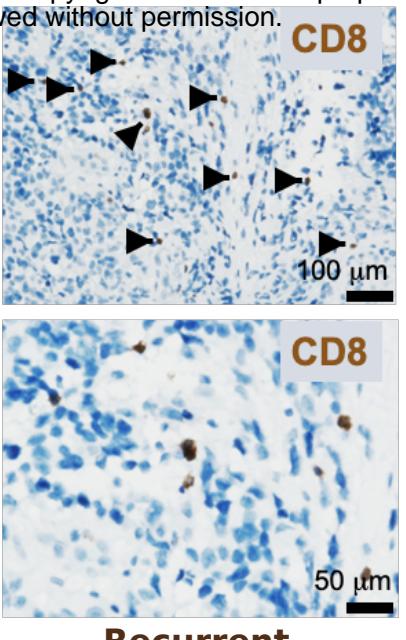

Recurrent

C

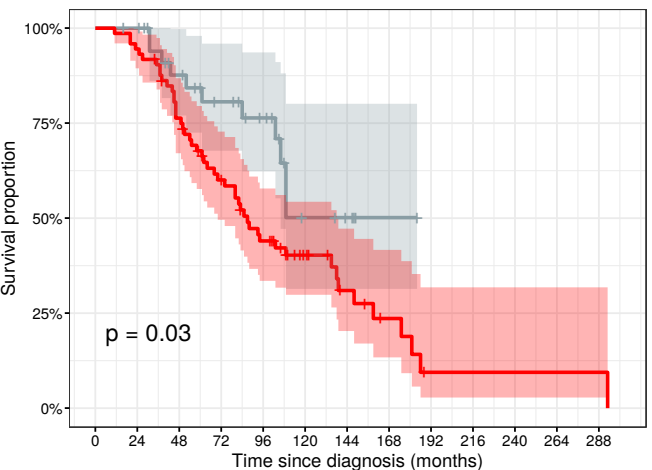

Number at risk

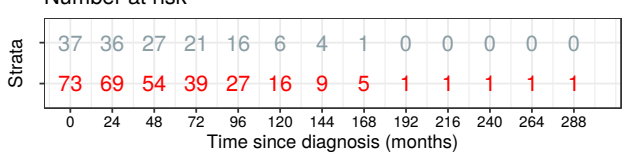

D

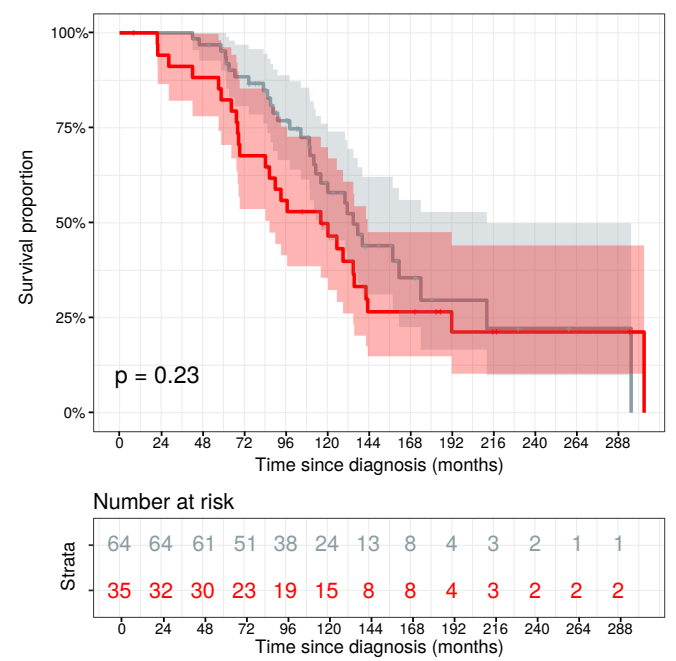

\section{GLASS-International}

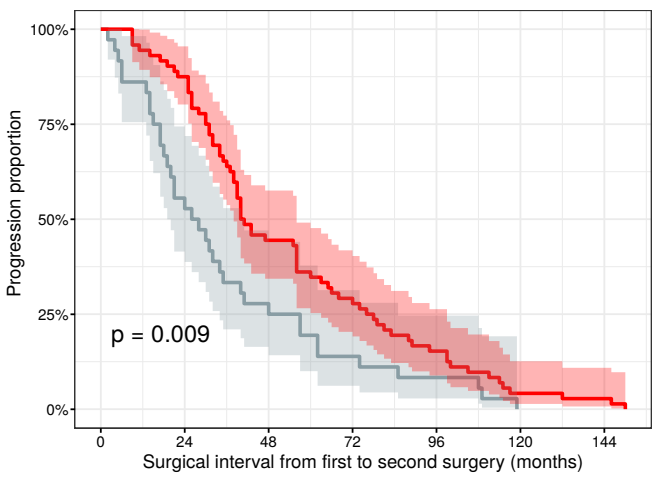

Number at risk
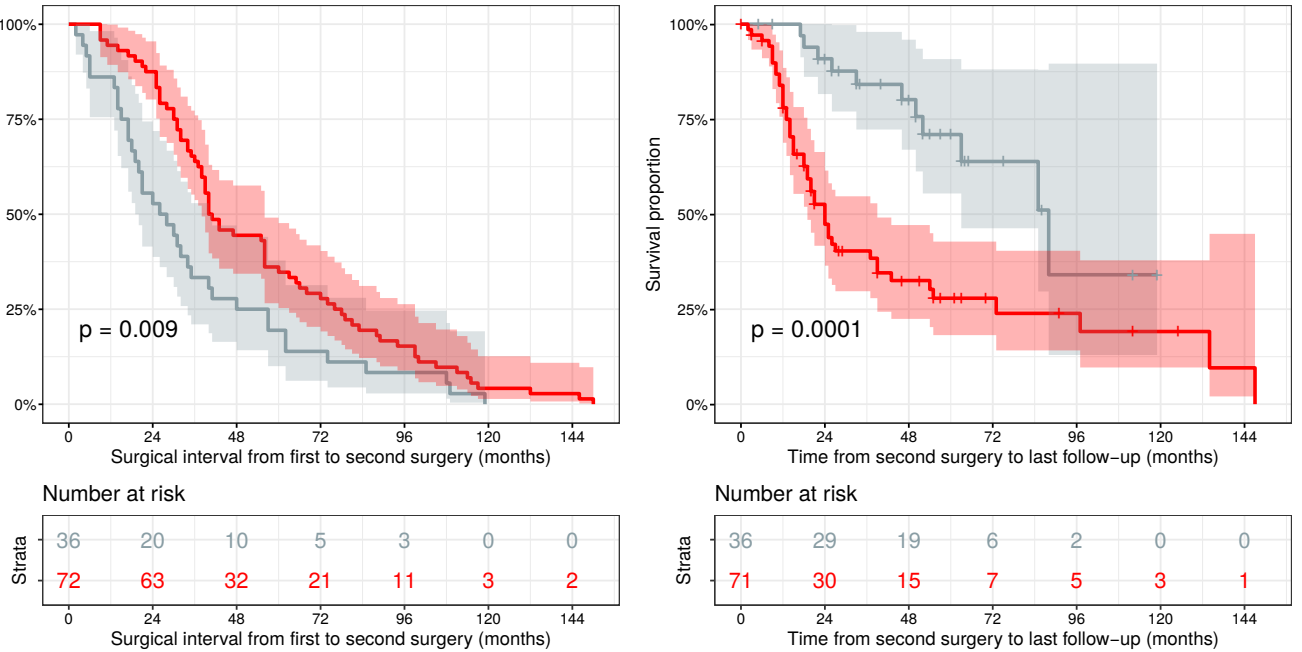

Number at risk

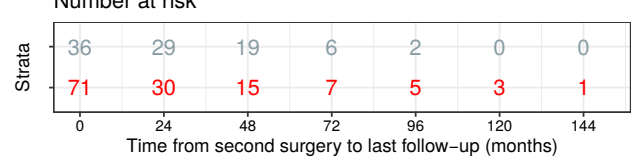

GLASS-NL

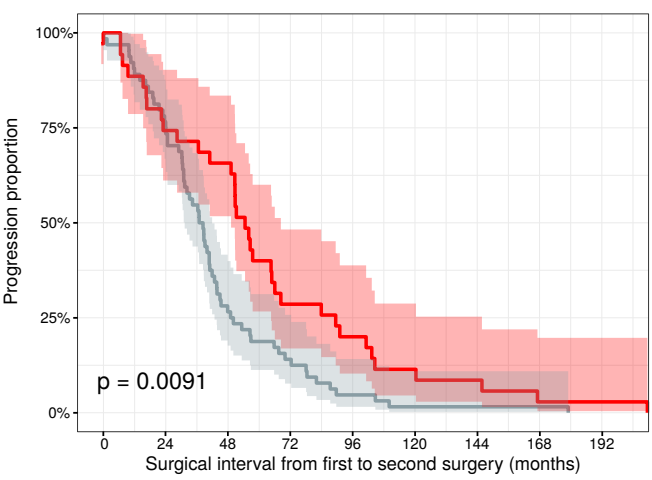

Number at risk

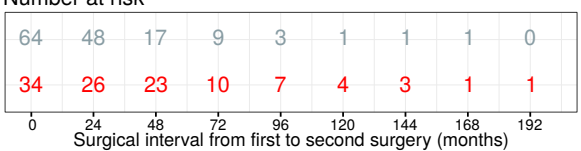

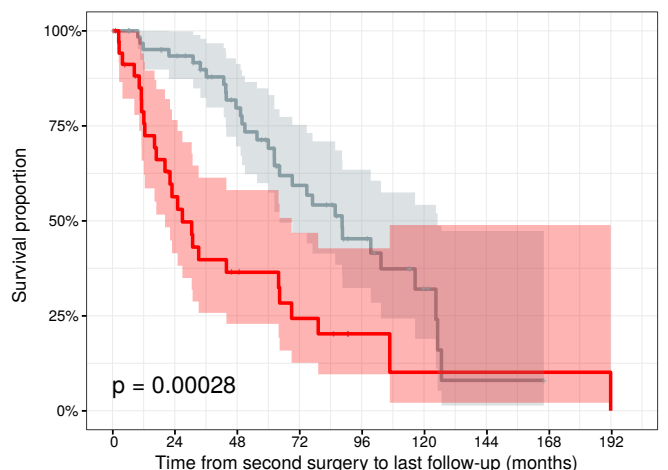

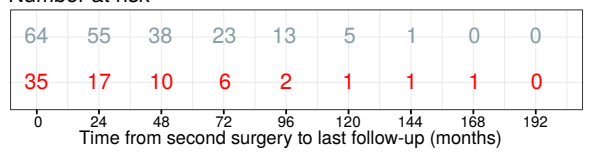

Number at risk

Treatment

Figure 4. Tumor microenvironment and clinical implications of treatment in IDH-mutant gliomas. A) CD31 and CD8 proportions (range scaled from 0 to 100\%) in samples originating from IDHmut matched initial and recurrent tumors in treated and non-treated patients. B) Illustrative immunohistochemical stainings for two marker proteins (CD31 and CD8) in an individual patient showing change of levels of tumor-infiltrating immune cells between initial and recurrent tumors. C/D) Overall survival and surgical interval analysis of IDHmut gliomas for the GLASS International (C) and GLASS-NL (D) cohorts. 


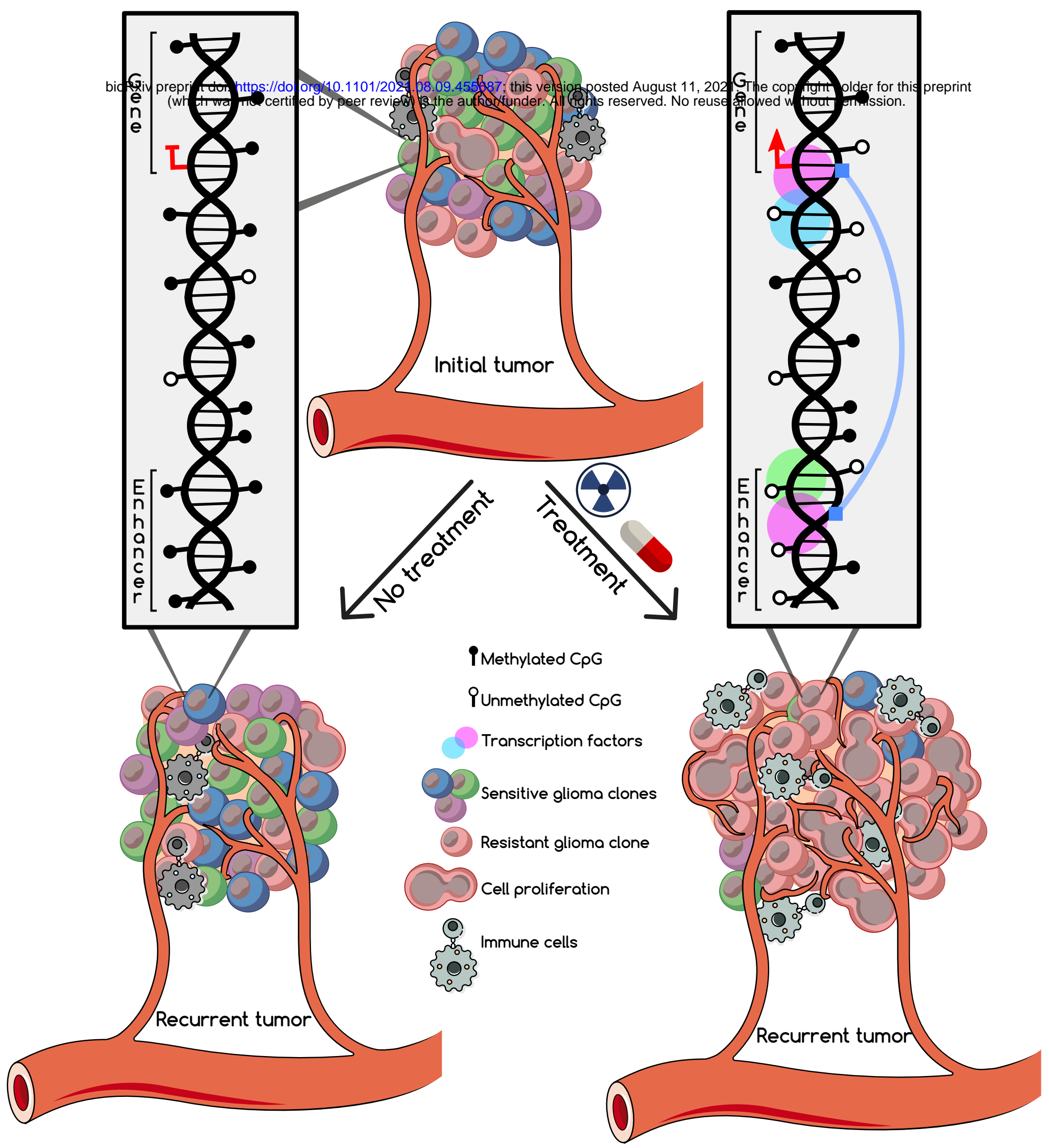

Figure 5. Schematic of treatment-induced epigenetic activation of cell cycle in IDHmut recurrent tumors. Standard treatment induces loss of methylation in IDHmut tumors with further epigenetic activation of cell cycle related genes associated with early tumor progression. In IDHmut non-treated patients, epigenetic changes are much more limited and tumors may progress later, either spontaneously or after subsequent treatment. 